Full Paper

\title{
FRUDISTOR: eine App zur Vorbeugung von Lagerungsverlusten
}

\author{
FRUDISTOR: an app to prevent storage disorders \\ FRUDISTOR: un'app per prevenire le perdite di prodotti in conservazione
}

\author{
Angelo Zanella ${ }^{1}$, Daniel A. Neuwald ${ }^{2}$, Andreas Bühlmann ${ }^{3}$, Ilaria Folie ${ }^{1}$, Dominikus Kittemann ${ }^{4}$, Nadine Klein ${ }^{2}$, Dirk Köpcke ${ }^{5}$, Cécile Prunier $^{2}$ \\ Oswald Rossi ${ }^{1}$, Barbara Stürz ${ }^{1}$, Elke Weinmann ${ }^{4}$, Daniel Würstl ${ }^{6}$ \\ ${ }^{1}$ Versuchszentrum Laimburg, Auer, Italien (ProjektPartner, mit Artikel Initiative) \\ 2 Kompetenzzentrum Obstbau Bodensee, Ravensburg, Deutschland (Projekt-Leiter) \\ ${ }^{3}$ Forschungsanstalt ACW Agroscope, Wädenswil, Schweiz \\ ${ }^{4}$ Hochschule Weihenstephan-Triesdorf, Weihenstephan, Deutschland \\ ${ }^{5}$ Esteburg - Obstbauzentrum, Jork, Deutschland \\ ${ }^{6}$ Internetagentur Bodensee, Ravensburg, Deutschland
}

\section{ABSTRACT}

Since physiological disorders and parasitical storage diseases of fruit in the postharvest chain of apple fruit cause sizable economic losses and waste valuable resources, there is an important need to correctly identify symptoms and to understand disorder development in order to put in place better strategies to prevent these disorders from occurring. For these reasons, an EU-funded INTERREG V-project "Alpenrhein, Bodensee, Hochrhein", started in 2015 for a three-year period. This project involves partners from different research institutions in Germany, Switzerland and Italy. Under the name Frudistor (the acronym of fruit disorders storage) an online platform for PCs and smart mobile devices has been generated on the site http://www.frudistor.de. The information is structured into descriptions of the origin, cause, prevention, susceptible cultivars, and disorders and diseases which can be easily confused with each other. Additionally, photos of symptoms are linked to the text. Several options to filter search results simplify disease and disorder identification. For example, the type of the symptom: internal and external, or time of appearance. Currently, Frudistor contains more than 40 disorders translated into Italian, German, Dutch, and English. The idea of creating an application accessible to all, free, dynamic, and expandable, was born from the desire to support users (producers, storekeepers, marketing, and consumers), making the process of harvest and storage increasingly more efficient and sustainable.

The following report collects a part of the result of this work, presenting some of the main damage that occurs during storage. The individual articles were written by the various authors who participated in the project and have been published in various journals in Italy, Germany, and Switzerland.

\section{KEYWORDS}

apple, storage disorder, storage disease, free sofware, updated photos

\section{CITE ARTICLE AS}

Zanella Angelo, Neuwald Daniel A., Bühlmann Andreas et.al. (2021) FRUDISTOR: an app to prevent storage disorders. Laimburg Journal 03/2021

DOI: $10.23796 /$ LJ/2021.001

\section{CORRESPONDING AUTHOR}

Angelo Zanella

Laimburg 6, Pfatten, 39040 Auer

(BZ), Italy

Angelo.Zanella@laimburg.it +390471969540 


\section{EINLEITUNG}

Diese Artikelsammlung ist ein Teil des Ergebnisses eines dreijährigen interregionalen EUProjekts im Rahmen des Interreg-V-Programm „Alpenrhein, Bodensee, Hochrhein" (2015-2018) finanziert und dank der Zusammenarbeit und der Beteiligung verschiedener Einrichtungen und Forschungszentren in Deutschland, Schweiz und Italien entwickelt worden. Dieses Projekt entspringt aus dem Wunsch heraus, zahlreiche häufige physiologische und parasitäre Schäden am Apfel zu verhindern und zu vermeiden, die sich trotz der stetigen Weiterentwicklung der modernsten Lagerungstechnologien entwickeln und während der Lagerung von Apfelfrüchten zu erheblichen wirtschaftlichen Verlusten führen können. Die beste Strategie, um dieser Situation entgegenzuwirken, besteht darin, dem Schaden vorzubeugen. Dazu werden alle erworbenen Kenntnisse und innovativen Strategien angewandt, um den optimalen Erntezeitpunkt zu ermitteln, um in der Folge die am geeignetsten Lagerungsbedingungen anwenden zu können. In diesem Sinne ist eine benutzerfreundliche und leicht verständliche Software-Applikation entwickelt worden: Frudistor (aus 'fruit disorders storage'). Diese Anwendung ist von jeglichem digitalen Endgerät aus unter http://www.frudistor.de zugänglich. Durch ein Filtersystem ist es möglich, den Schadenstyp am Apfel, den Schadensort und das zeitliche Auftreten auszuwählen und in der Folge das jeweilige technische Datenblatt, welches detaillierte Informationen über mögliche Ursachen, Symptome und Präventionsstrategien liefert, zu lesen. Eine Fotoserie, welche die verschiedenen Krankheiten und deren Entwicklungsstadien darstellt, ermöglicht es, einen ikonografischen Vergleich anzustellen. Auf diese Weise kann der Anwender die verschiedenen am Apfel beobachteten Schäden vergleichen und dadurch die Schadbezeichnung und die möglichen Ursachen herausfinden. Zurzeit beinhaltet Frudistor mehr als 40 technische Datenblätter in deutscher, italienischer, niederländischer und englischer Sprache. Die Idee, eine Anwendung zu entwickeln, die für alle zugänglich, kostenlos, dynamisch und erweiterbar ist, entstand aus dem Wunsch heraus, die verschiedenen Anwender des Sektors (Produzenten, Lagertechniker und Handelsorganisationen) und die Konsumenten zu unterstützen, um den Prozess der Ernte und Lagerung noch effizienter und nachhaltiger zu gestalten.

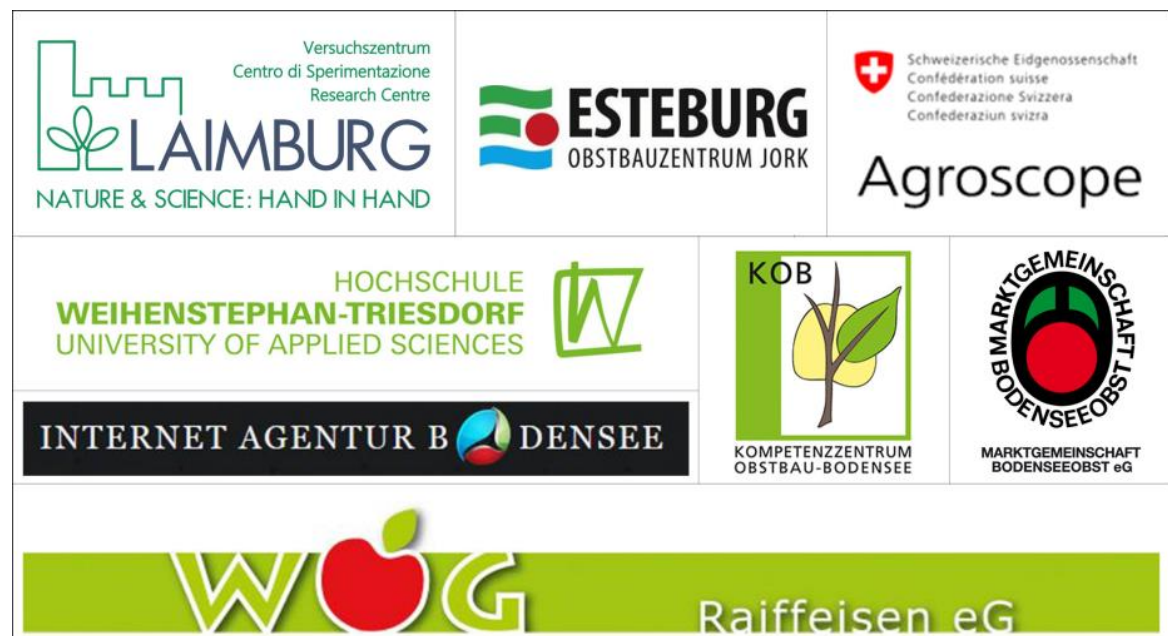

Württembergische Obstgenossenschaft Raiffeisen e.G.

Der folgende Artikel will die Erkenntnisse dieses Projekts sammeln und stellt die wichtigsten Schäden vor, die während der Lagerung von Äpfeln auftreten können, wie z.B. Kernhausbräune, Schalenbräune, Glasigkeit, Schrumpfen, Schalenflecken, Druckstellen, Weiche Schalenbräune, Trübschaligkeit, Alters-Fleischbräune, Innere Fleischbräune (CA-bedingt), Aufspringen, Schalenverätzungen und Stippigkeit. Die einzelnen Beiträge wurden von den verschiedenen Autoren, die am Projekt beteiligt waren, verfasst und in verschiedenen Zeitschriften in Italien, Deutschland und der Schweiz veröffentlicht.

\section{PROJEKT TEILNEHMER:}

- Versuchszentrum Laimburg, Auer, Italien

- Kompetenzzentrum Obstbau Bodensee, Ravensburg, Deutschland

- Hochschule Weihenstephan-Triesdorf, Weihenstephan, Deutschland

- Esteburg Obstbauzentrum Jork, Deutschland

- Agroscope, Wädenswil, Schweiz

- Internetagentur Bodensee, Ravensburg, Deutschland

- Marktgemeinschaft Bodenseeobst eG, Friedrichshafen, Deutschland

- Württembergische Obstgenossenschaft Raiffeisen eG, Ravensburg, Deutschland

\section{KERNHAUSBRÄUNE - VOM DUNKLEN HERZ DER ÄPFEL [1] [2] [3] [4] [5] [6]}

\section{SCHADBILD}

Kernhausbräune ist nicht zu verwechseln mit Kernhausfäule (Abb. 1 a). Beide Schäden sind auf das Kernhaus beschränkt, jedoch ist die Kernhausbräune rein physiologisch bedingt. Sie erscheint als eine Verbräunung des Fruchtfleisches, welches an das Kernhaus anschließt. Betroffen ist immer das Markgewebe zwischen den Samenfächern von wo aus sich die Schädigung bis zu den Leitbündeln ziehen kann. Bei altersbedingter Kernhausbräune (Abb. 1 b, c) erscheint eine diffuse Verbräunung gegen Ende der Lagerdauer oder bei nicht optimalen Lagerbedingungen. Das betroffene Gewebe ist mehlig trocken, es berührt nur wenig die Samenfächer, wohingegen Kernhausbräune auf Grund eines CA-Schadens (Abb. $1 \mathrm{~d}$, e) schon nach kurzer Zeit mit einer intensiven, klar abgegrenzten Verbräunung zu sehen ist. Bei besonders schwerer Ausprägung von Kernhausbräune können sich im geschädigten Fruchtfleisch Kavernen bilden (Abb. $1 \mathrm{f}$ ). Sie bilden sich durch das Absterben von Zellen. Der Zellsaft tritt aus den geschädigten Zellen aus und wird von den umliegenden Zellen aufgenommen, zurück bleibt dann ein Hohlraum.

\section{PHYSIOLOGISCHER HINTERGRUND}

Die Entstehung von Kernhausbräune ähnelt der von Fleischbräune. Sie kann zwei unterschiedliche Ursachen haben. Zum ersten 

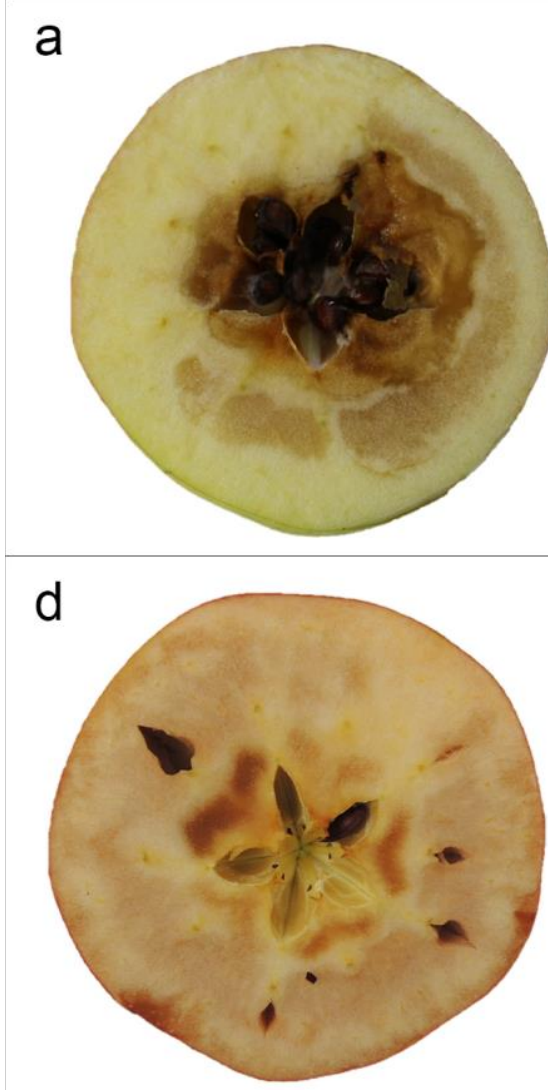

b

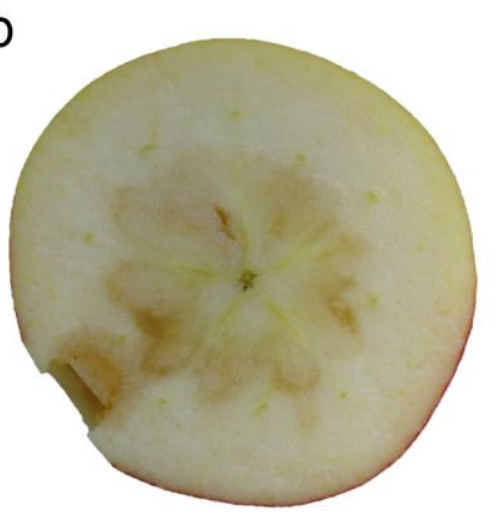

e

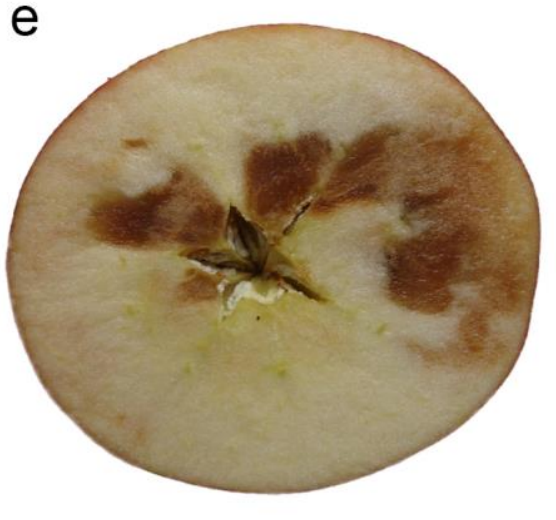

C

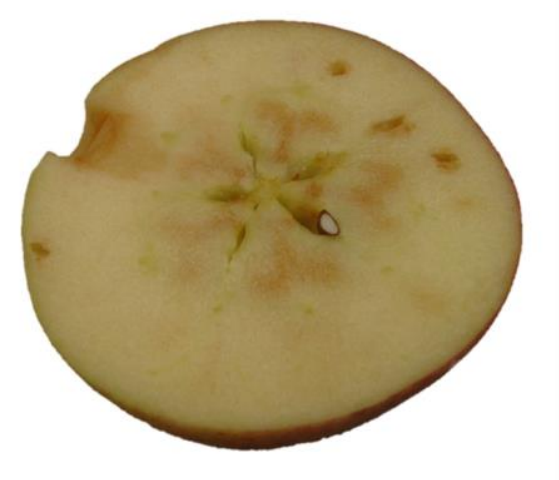

f

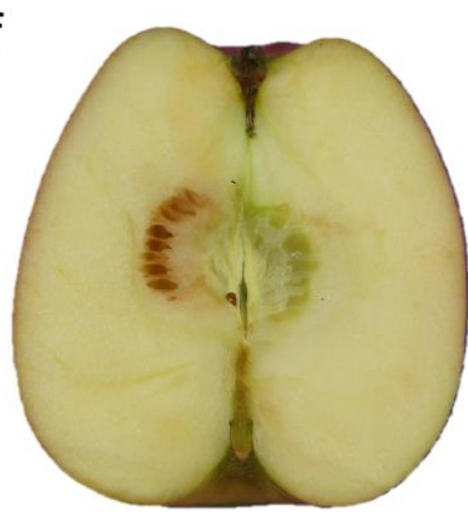

Abb. 1: Kernhausbräune // Core browning.

entsteht sie bei Überalterung der Früchte durch zu lange Lagerung, ungünstige Lagerbedingungen oder sie entwickelt sich wegen der Einlagerung zu reifer Früchte. Die zweite Möglichkeit können auch ungünstige CAEinstellungen sein, in diesem Fall zu geringe O2- oder zu hohe CO2- Gehalte in der Lageratmosphäre. Sie kann deshalb sowohl nach kurzer (CA-bedingte Kernhausbräune) sowie nach längerer (altersbedingte Kernhausbräune) Lagerperiode auftreten.

Die Alterung der Früchte wird außerdem durch wechselnde Lagerbedingungen mit Phasen erhöhter Atmung besonders gefördert. Das kann durch unbeabsichtigte Temperaturerhöhung, zu viel $\mathrm{O} 2$ oder zu wenig $\mathrm{CO} 2$ in der Lageratmosphäre passieren. Deshalb sollte darauf geachtet werden, während der gesamten Lagerdauer die gleichen, einmal optimal eingestellten Bedingungen beizubehalten.

Bei übergroßen Früchten können diese Symptome durch eine erschwerte Gasdiffusion im Gewebe noch verstärkt werden. Schon nach kurzer Zeit können kleine Teile des Kernhauses geschädigt werden. In diesem Bereich sterben die Zellen ab und es besteht erhöhte Gefahr der Kavernenbildung.
Obwohl die Ursachen von Kernhausbräune durch physiologische Prozesse im Apfel entstehen, gibt es auch sortentypische Unterschiede in der Anfälligkeit. So sind Braeburn und Topaz bekannt für die Ausbildung beider Arten von Kernhausbräune. Boskoop, Gloster und Jonagold gelten dagegen als empfindlich für altersbedingte, Nicoter bzw. Kanzi $^{\circledR}$ eher für CA-bedingte Kernhausbräune.

\section{VORBEUGUNG UND MAßNAHMEN}

Ist das Schadbild einmal entstanden lässt es sich nicht wieder rückgängig machen. Aus diesem Grund sollte besonders auf die Vorbeugung geachtet werden:

In der Anlage:

- Früchte von Bäumen mit gleichmäßigem, mittlerem Behang;

- ruhiges Baumwachstum;

- gleichzeitiges Ernten von Partien, die zusammen gelagert werden, optimale Erntezeitpunkte auswählen.

Im Lager:

- Auswahl geeigneter Lagerbedingung für jede Sorte, sowie rechtzeitige und konsequente Einstellung;
- Lagerdauer der Fruchtqualität anpassen

- O2 möglichst im ULO bzw. DCA-Bereich und optimale $\mathrm{CO}$-Konzentration einstellen.

\section{SCHALENBRÄUNE - WENN DIE FRUCHTSCHALE DER ÄPFEL IM LAGER VERBRÄUNT [7] [8] [9] [10] [11] [12]}

\section{SCHADBILD}

Die Fruchtschale von Äpfeln kann im Lager oberflächliche Verbräunungen der Epidermis aufweisen, welche meist nicht auf das darunterliegende Gewebe übergehen. Diese Verbräunungen sind auf Stoffwechselstörungen, sogenannte physiologische Störungen der Frucht zurückzuführen. Grundsätzlich können verschiedene SchalenbräuneArten unter Einbezug des Reifestadiums der Frucht, der Fruchtstellen, an denen sich die Symptome äußern, und der Kombination mit anderen Schäden, unterschieden werden, wobei die Schadensbilder der verschiedenen Schalenbräune-Typen optisch oft nicht klar unterscheidbar sind. „Gewöhnliche Schalenbräune" (Abb. 2 a) entwickelt 


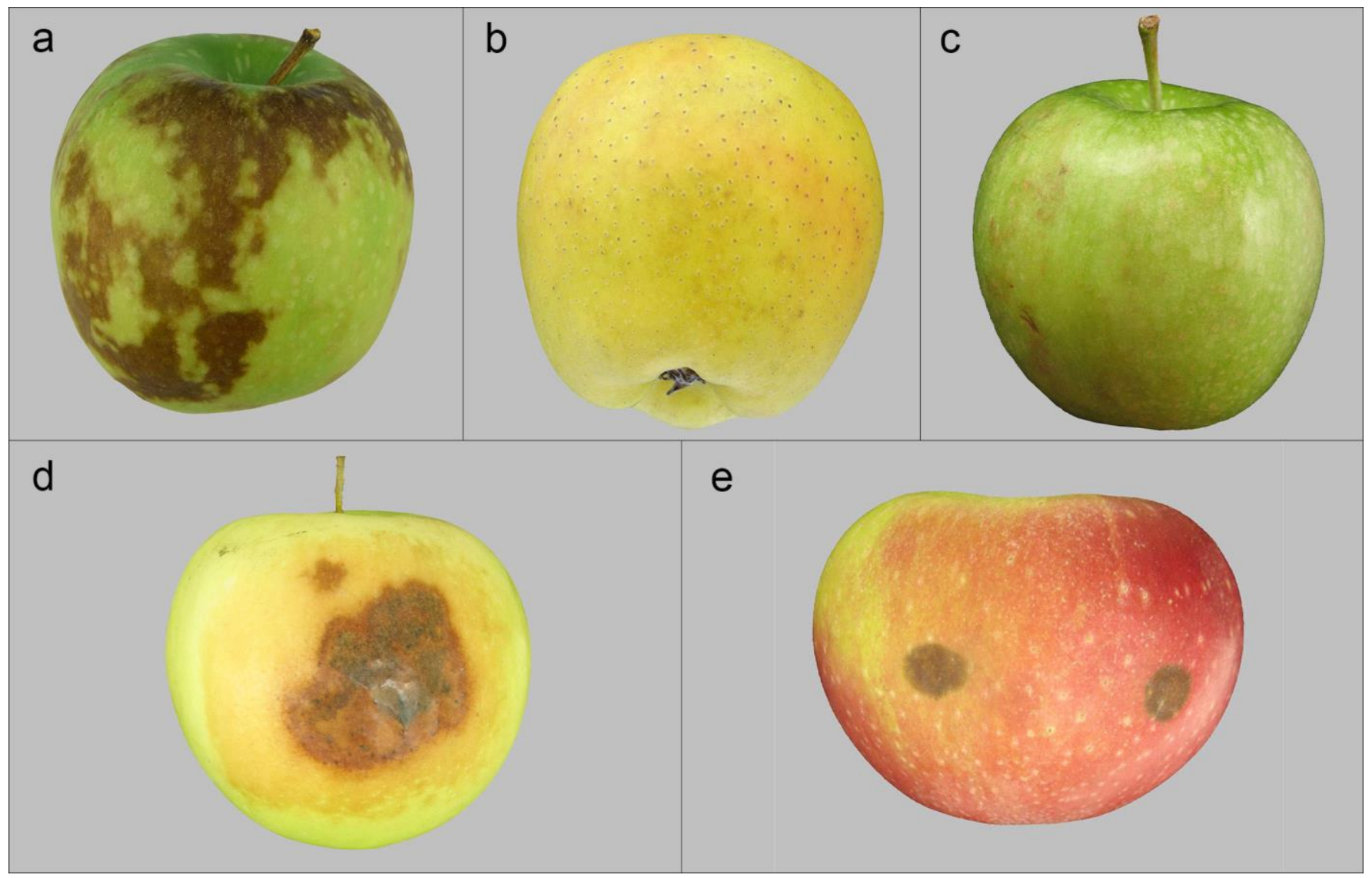

Abb. 2: Schalenbräune // Superficial scald.

sich während der Lagerung und vor allem während der Nachlagerung (Shelf-Life) der Früchte.

Die oberflächlichen, meist unscharf begrenzten Verbräunungen der Schale gehen nicht auf das darunterliegende Fruchtfleisch über. Zudem kann an den betroffenen Stellen die Fruchtschale etwas einsinken. Nach der Entnahme der Früchte aus dem Lager kann es bei Raumtemperatur zu einer schnellen Ausbreitung der Symptome kommen. Gewöhnliche Schalenbräune tritt vornehmlich, aber nicht ausschließlich, bei früh geernteten Früchten spezifischer Sorten, wie z.B. Granny Smith oder Red Delicious, vorwiegend an deren Schattenseite auf und wird durch hohe Temperaturen vor der Ernte zusätzlich gefördert. „Altersschalenbräune" (Abb. 2 b) unterscheidet sich vom Schadbild her nicht wesentlich von jenem der gewöhnlichen Schalenbräune: die Schale der Früchte weist eine oberflächliche Bräunung auf, nach längerer Lagerung sinkt das Gewebe an den betroffenen Stellen etwas ein, jedoch kann manchmal auch das darunterliegende Fruchtfleisch verbräunen. Im Gegensatz zur gewöhnlichen Schalenbräune treten die Symptome nicht nur an der Schattenseite, sondern häufig auf der Sonnenseite von zu spät geernteten Früchten auf. Altersschalenbräune tritt bei vielen Apfelsorten auf, z.B. Golden Delicious, meist wegen zu später Ernte und sich daraus ergebender Überlagerung. Die „Diffuse Schalenbräune" könnte mit der gewöhnlichen Schalenbräune verwechselt werden. Das Schadbild (Abb. 2 c) zeigt diffuse, oberflächliche, durch die Fruchtschale durchscheinende Verbräunungen und zusätzlich kann die Schale an den betroffenen Stellen rau werden. Es kommt jedoch zu keiner Einsenkung des Fruchtfleisches an den betroffenen Stellen, wie es bei der gewöhnlichen Schalenbräune der Fall ist. Das Auftreten von diffuser Schalenbräune wird, im Gegensatz zur gewöhnlichen Schalenbräune, durch Nachernte-Behandlungen mit dem Reifehemmstoff 1-Methylcyclopropen (1-MCP) verstärkt. Erste Symptome der genannten Störung treten häufig schon nach 2 Wochen im Kühllager auf und gegen Ende der Lagersaison kann das Schadausmaß hoch sein. Diffuse Schalenbräune stellt vor allem in Produktionsgebieten, in welchen heiße Sommer und wenig Regenfall vorherrschen, ein Problem dar. Die "Sonnen-Schalenbräune“ entwickelt sich im Lager meist auf Früchten, die im Feld plötzlich intensiv der Sonne ausgesetzt wurden. Durch starken Sommerschnitt oder Auspflücken, beispielsweise, können Schattenfrüchte direkt der Sonne ausgesetzt werden und sind daraufhin sehr empfindlich gegenüber Sonnenschäden. Während die Symptome eines Sonnenbrandes bereits zur Ernte sichtbar sind und sich als rötlich-braune Verbrennungen an der sonnenexponierten Seite der Frucht zeigen, entwickelt sich Sonnen-Schalenbräune erst während der Lagerung. Die Symptome (Abb. 2 d) äußern sich als sehr dunkle, braune, Flecken, welche sich ausschließlich auf der plötzlich sonnenexponierten Seite der Frucht ausbilden. Das Auftreten der sogenannten "Kontaktschalenbräune" (Abb. 2 e) wurde bisher an der Sorte Stayman Winesap beobachtet. Die Symptome entwickeln sich im Lager als oberflächliche, kreisrunde Verbräunungen an den Kontaktstellen der Früchte.

\section{URSACHE UND PHYSIOLOGISCHER HINTERGRUND}

Die Entstehung der verschiedenen Schalenbräune-Arten ist auf unterschiedliche Ursachen zurückzuführen. Während man an- 
nimmt, dass die Induktion für die Entwicklung der gewöhnlichen Schalenbräune auf den Kälteschock („Chilling“) zu Beginn der Lagerung zurückzuführen ist und hauptsächlich früh geerntete Früchte für obgenannten Schaden anfällig sind, wird Altersschalenbräune durch zu spätes Ernten oder durch eine Überlagerung der Früchte gefördert und ist somit eng mit der Seneszenz (Alterung) und der Lagerdauer der Früchte in Verbindung zu setzen. Die Tatsache, dass diffuse Schalenbräune auf Früchten warmer Anbaugebiete, welche mit 1-MCP behandelt worden sind, gefördert wird, zeigt, dass der Entstehung dieser Art der Schalenbräune eine andere Ursache zu Grunde liegt. Die Entwicklung und der Zeitpunkt des Auftretens von Sonnen-Schalenbräune im Lager hingegen stehen in direktem Zusammenhang mit dem Ausmaß der Sonnenintensität im Feld.

Die genauen Ursachen für das Auftreten von Kontaktschalenbräune sind bis dato nicht abgeklärt. Erste Untersuchungen haben jedoch ergeben, dass das Auftreten von Kontaktschalenbräune im Lager bei der Sorte Stayman Winesap mit der Reife der Früchte zusammenhängt; vor allem zu spät geerntet Früchte neigen bei ungünstigen Lagerbedingungen dazu, den genannten Schaden zu entwickeln.

Nennenswert sind auch die sortentypischen Unterschiede in der Anfälligkeit für die verschiedenen Schalenbräune-Arten: So gelten Granny Smith und Red Delicious als besonders anfällig für die Ausbildung von gewöhnlicher Schalenbräune, während Golden Delicious, Jonagold und Idared vor allem für Alters-Schalenbräune anfällig sind. Sowohl diffuse Schalenbräune als auch Sonnen-Schalenbräune stellen vor allem bei Golden Delicious und Granny Smith ein Problem dar. Die verschiedenen Schalenbräune-Arten können jedoch auch bei anderen Sorten als den hier aufgelisteten, auftreten.

\section{VORBEUGUNG UND MAßNAHMEN}

Als Vorbeugungsstrategie der verschiedenen Schalenbräune-Arten wird eine Kombination aus kulturtechnischen Maßnahmen im Feld und einer optimalen Lagerung empfohlen: Grundsätzlich kann das Auftreten von gewöhnlicher- und Altersschalenbräune durch eine termingerechte Ernte, angepasst an die Lagerdauer und an die Lagertechnologie, ein effizientes Einlagern der Früchte und eine Lagerung bei niedrigen Sauerstoffwerten vermieden werden. Eine Lagerung der Früchte unter ULO- Bedingungen (O2: 1$1,5 \%)$ ermöglicht es, die gewöhnliche Schalenbräune stark zu verzögern oder zu verhindern. Zusätzlich kann eine Nacherntebehandlung mit 1-MCP helfen, der gewöhnlichen Schalenbräune entgegenzuwirken. Im Gegensatz dazu, können Nacherntebehandlungen mit 1-MCP eine unzureichende Wirkung zur Vorbeugung von Alters-Schalenbräune, im Falle von Überreife, haben. Eine Alternative, um die Schadensentwicklung der gewöhnlichen Schalenbräune bei stark anfälligen Sorten zu minimieren, besteht in der Lagerung der Früchte unter dynamisch kontrollierter Atmosphäre (DCA) bei $\mathrm{O2}$ : 0,4-0,7\%. Zudem sollten die Kulturmaßnahmen im Feld auf die Erziehung von Bäumen mit gleichmäßig belichteten Früchten abzielen. Die diffuse Schalenbräune kann nur durch eine optimale Temperaturführung (Stufenkühlung) und eine verzögerte Applikation von 1-MCP kontrolliert werden, während als Vorbeugungsmaßnahme von Sonnen-Schalenbräune eine sorgfältige Sortenauswahl und der Einsatz von Hagelnetzen empfohlen wird, welche das Risiko eines Sonnenbrandes, und infolgedessen auch das mögliche Auftreten einer Sonnen-Schalenbräune während der Lagerung verringern. Nacherntebehandlungen mit 1-MCP zeigen keine Wirkung zur Vorbeugung von SonnenSchalenbräune. Kontaktschalenbräune kann durch eine termingerechte Ernte der Früchte vermieden werden; weiters haben erste Untersuchungen gezeigt, dass eine Lagerung der Früchte unter dynamisch kontrollierter Atmosphäre die Entwicklung von Kontaktschalenbräune bei der Sorte Stayman Winesap größtenteils verhindern konnte.

\section{GLASIGKEIT - DES EINEN SEGEN IST DES ANDEREN FLUCH [13] [14] [15] [16] [17] [18] \\ SCHADBILD UND URSACHE}

Glasigkeit oder auch Wassersucht genannt, ist eine physiologische Störung im Zuckerstoffwechsel der Frucht, deren Symptome in Ländern wie Brasilien oder Japan Qualitätsmerkmale darstellen. In Europa jedoch ist stark ausgeprägte Glasigkeit ein Grund für Reklamationen und unter Umständen auch für eine eingeschränkte Lagerfähigkeit. Sehr empfindliche Sorten sind unter anderem Fuji (Abb. 3 a, b), Mairac (Abb. 3 c), Cox Orange, Gloster und Alkmene. Teilweise bzw. weniger empfindlich sind Golden Delicious,
Boskoop, Jonagold, Braeburn (Abb. 3 d), Cameo, Gala, Delbar Estivale, Kanzi ${ }^{\circledR}$-Nicoter und Pinova.

\section{PHYSIOLOGISCHER HINTERGRUND}

Wie der Name schon vermuten lässt, entstehen bei der Glasigkeit, je nach Ausprägungsgrad, glasig durchscheinende Stellen in der Frucht (Abb. 3 e). Bei Fuji finden sich diese zunächst im Bereich der Gefäßbündel und wandern mit zunehmender Ausprägung Richtung Kernhaus. Bei anderen Sorten wandern die glasigen Stellen meist sternförmig nach außen und umfassen später flächig ganze Teile des Fruchtfleisches, oder bilden sich gar zuerst unter der Schale. Die Symptome können bereits am Baum auftreten und bei betroffenen Früchten unter Umständen zu einem Gärgeschmack führen. Begünstigt wird das Auftreten von Glasigkeit durch sehr günstige Photosynthese-Bedingungen vor der Ernte, also starker Sonneneinstrahlung und hohen Tagestemperaturen (über $30{ }^{\circ} \mathrm{C}$ ), sowie kalten Nachttemperaturen. Normalerweise wird im Zuge der Photosynthese Energie in Form von Kohlenhydraten (Zucker) in die Frucht eingelagert, welche später im Lager als Energielieferant zum Aufrechterhalten der physiologischen Prozesse in der Frucht dienen. Da es bei sehr günstigen Photosynthese-Bedingungen zu einer verstärkten Zuckerproduktion in den Blättern und nachts zu einem niedrigeren Zuckerverbrauch beim Atmungsprozess kommt, entsteht ein Zuckerüberschuss. Der Zucker wird in seiner Transportform Sorbitol von den Blättern in die Früchte transportiert, staut sich dort in den Zellzwischenräumen, welche unter normalen Umständen mit Luft gefüllt sind, und verursacht so die glasigen Stellen. Zusätzlich wird, durch einen Mangel an Calcium, das osmotische Potential im Zellzwischenraum reduziert. Dies führt zu einem Austritt von Wasser aus den Zellen in die Interzellularen. Zu erwähnen ist hier auch der Einfluss des Erntezeitpunktes. Durch den fortgeschrittenen Stärkeabbau sehr reifer Früchte wird mehr Zucker zur Verfügung gestellt, welchen die Zellen nicht mehr aufnehmen können, was das Auftreten von Glasigkeit verstärkt.

Bei der Einlagerung kann eine schnelle Überführung der Äpfel ins CA-Lager ebenfalls eine verstärkte Bildung von Fleischbräune und Gärgeschmack hervorrufen, da die niedrigen O2-Werte bzw. zu hohen CO2-Werte zu einem zusätzlichen Sauerstoffmangel in den bereits mit Zellsaft gefüllten Zellzwischenräumen führen können. Der Abbau 

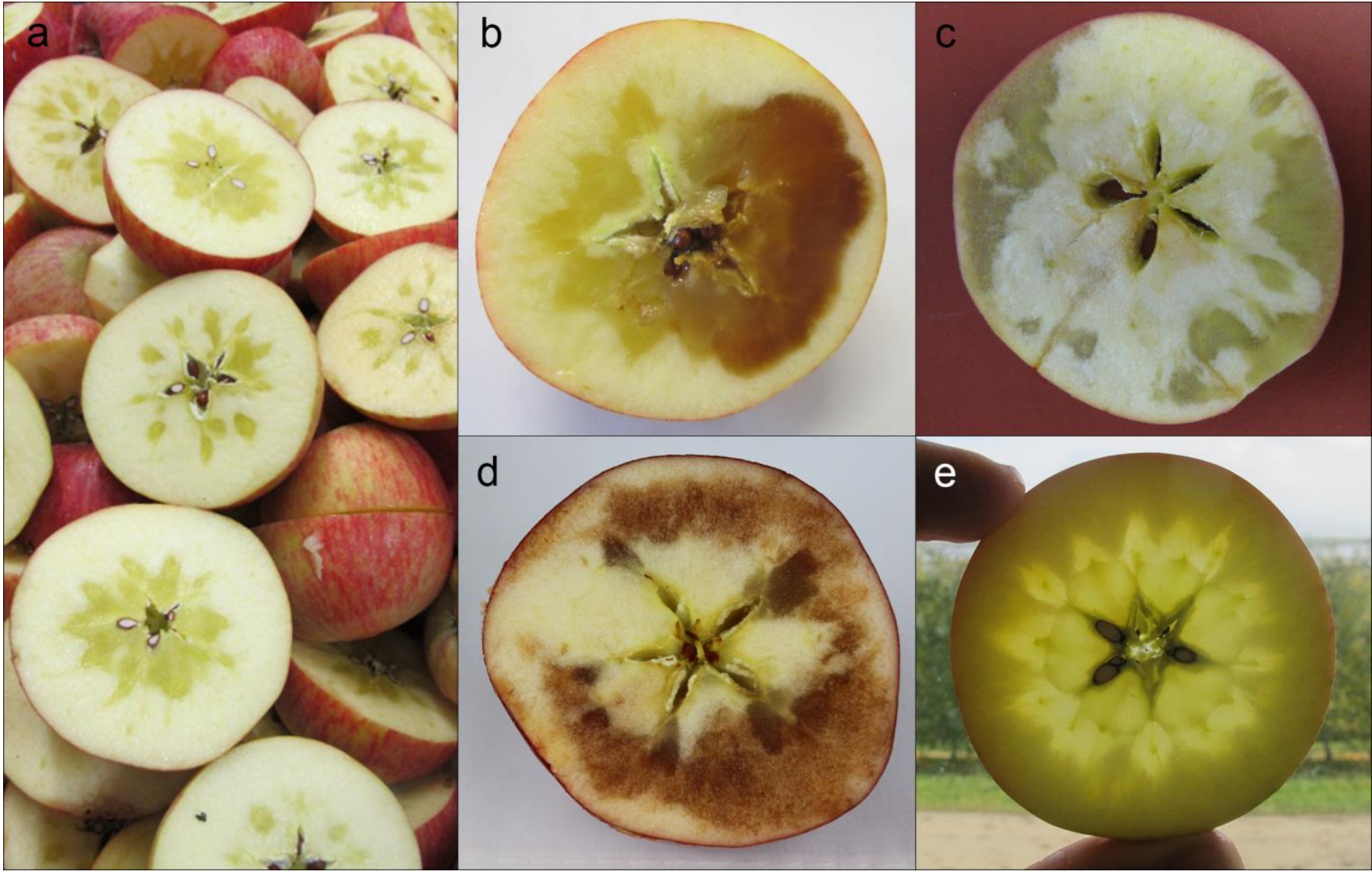

Abb. 3: Glasigkeit // Watercore.

der Glasigkeit ist möglich, wobei die Temperatur und eventuell die Transpiration eine entscheidende Rolle spielen.

\section{VORBEUGUNG UND MAßNAHMEN}

- Vorlagerung der Äpfel im Kühllager bei 3$4{ }^{\circ} \mathrm{C}$ für 6 Wochen inklusive regelmäßiger Fruchtkontrollen zur Überprüfung des Abbaus der Glasigkeit

- 1-MCP Einsatz (Stabilisierung der Fruchtfestigkeit)

- Ausgeglichenes Blatt-/ Frucht-Verhältnis

- Vermeiden von geringem Fruchtbehang, spätem Erntetermin, sowie Calcium-Mangel

- Wahl geeigneter Lagermaßnahmen (höhere Lagertemperatur)

- Ein optimaler Erntezeitpunkt ist die beste Strategie zur Vermeidung von Glasigkeit.

\section{SCHRUMPFEN (WELKEN) VON ÄPFELN IM LAGER [19] [20] [21] [22] [23] [24]}

\section{SCHADBILD}

Die Luftfeuchtigkeit stellt bei der Lagerung einen der wichtigsten Faktoren dar, da sie zum einen den Wasser- und damit Gewichtsverlust der Frucht beeinflusst, zum anderen, da sie das Auftreten physiologischer Lagerkrankheiten sowohl positiv als auch negativ beeinflussen kann. Dabei ist es jedoch nicht das Ziel, den Wasserverlust der Frucht gegen Null zu reduzieren. Ein gewisser Gewichtsverlust der Früchte (ca. 2-3\%) ist sogar gewünscht, da dadurch das Risiko für bestimmte physiologische Lagerkrankheiten, wie der Schalenbräune, reduziert werden kann. In der Regel werden bei Kernobst sortenabhängig ab einem Massenschwund von 4-6\% Schrumpfungserscheinungen sichtbar.

\section{SYMPTOME}

Die Schale der betroffenen Früchte ist nicht mehr straff und elastisch. Sie zeigt Falten und der gesamte Apfel ist etwas weicher. Beim Öffnen der Frucht fehlt die Knackigkeit und das Fruchtfleisch ist weniger saftig (Abb. 4 a, b). Verbräunungen des Fruchtfleisches werden in der Regel durch den Wasserverlust nicht verursacht.

\section{PHYSIOLOGISCHER HINTERGRUND}

Das Schrumpfen der Früchte wird durch eine zu niedrige relative Luftfeuchte und einen damit verbundenen, verstärkten Wasserverlust der Früchte verursacht.

Eine relative Luftfeuchtigkeit im Kernobstlager zwischen $92 \%$ und $95 \%$ gilt als optimal. $\mathrm{Ab}$ Werten über $98 \%$ geben die Früchte in der Regel kein Wasser mehr ab (Gleichgewicht zwischen der Wasserhaltekraft der Frucht und dem Aufnahmevermögen der Luft), jedoch steigt bei zu hoher Luftfeuchte das Risiko für pilzliche Fruchterkrankungen sowie Schimmelbildung an den Kisten und Fruchtstielen. Des Weiteren kann das Auftreten bestimmter physiologischer Lagerschäden, wie der Schalenbräune, bei zu hoher Luftfeuchte begünstigt werden. Letzteres ist vor allem darauf zurückzuführen, dass sich bei fehlender Wasserabgabe für die Frucht schädliche Verbindungen ( $\alpha$-Farnesen) auf der Fruchtoberfläche konzentrieren und zu Verbräunungen der Schale führen können. Deshalb ist ein gewisser Gewichtsverlust der Früchte (ca. 0,3\% pro Monat) erwünscht. Erfahrungen haben gezeigt, dass die Empfindlichkeit für Schalenbräune in südlicheren Anbauregionen in der Regel höher ist. Der größte Wasserverlust im Lager erfolgt vor allem zum Zeitpunkt der Einlagerung, da hier bedingt durch den Abkühlpro- 
zess der warmen Früchte die Kälteanlagen sehr lange kühlen. Wenn die Kälteanlage läuft, liegt die Verdampfer-Temperatur im Lager tiefer als die Raumtemperatur. An den Lamellen des Verdampfers kommt es dadurch zur Taupunktunterschreitung und somit zur Bildung von Kondenswasser. Um das dadurch entstandene Sättigungsdefizit der entfeuchteten Luft auszugleichen, wird den Früchten Wasser entzogen. Dies erklärt auch, warum in einem Raum mit großem Leervolumen (größeres Sättigungsdefizit) die Früchte in der Regel deutlich mehr Wasser verlieren. Je länger bzw. öfter die Kälteanlage läuft, umso größer ist die Entfeuchtung der Früchte.

\section{VORBEUGUNG}

- Dimensionierung und Einstellung der Kälteanlage. Da früher die Lagertemperatur in der Regel höher war als heute, lässt sich in alten Räumen häufig eine zu geringe Dimensionierung der Verdampferoberfläche finden. Dies bedeutet, dass bei niedrigerer Solltemperatur die Anlage entsprechend länger läuft, um die Sollwerte zu halten, wodurch die Entfeuchtung der Früchte steigt. Ein weiterer Grund kann eine zu enge Hysterese, also Einstellung der Minimal- und Maximal-Werte, bei der Steuerung der Kälteanlage sein. Dadurch erhöht sich die Anzahl der Kühltakte, wodurch ständig Wasser am Verdampfer auskondensiert. Auch eine zu tief eingestellte Verdampfungstemperatur kann Ursache für einen erhöhten Wasserverlust sein.

- Mangelnde Isolierung, schlechte Stapelung, defekte Verdampfer. Mangelnde Isolierung der Wände, Türen oder des Bodens kann zudem eine erhöhte Entfeuchtung der Früchte begünstigen, da sich durch den Wärmeeintrag die Laufzeit der Anlage verlängert. Außerdem führt eine zu enge Stapelung der Großkisten bzw. ein Nicht-Einhalten von Stapelabständen zu einer reduzierten Effektivität der Kälteanlage, da die Abfuhr der Wärme von der Lagerware zum Verdampfer erschwert wird. Dies führt zum einen wiederum zu längeren Laufzeiten der Anlage, zum anderen bilden sich in Bereichen mit schlechter Luftführung Wärmenester, an denen auf Grund des höheren Sättigungsdefizits die Transpiration zunimmt. Auch defekte Verdampfer oder Ventilatoren können die Laufzeit der Kälteanlage und damit den Wasserverlust der Früchte deutlich erhöhen. Alle genannten Faktoren führen zu einer längeren Laufzeit der Kälteanlage und damit zu einem größeren Gewichtsverlust der Früchte. Als Konsequenz der Bildung von Kondenswasser an den Verdampfern kommt es häufig zum Vereisen der Lamellen, wodurch der Wirkungsgrad der Anlage abnimmt bzw. die Laufzeiten zunehmen.

- Einfluss von Sorte und Erntetermin. Der Wasserverlust der Früchte kann zusätzlich von weiteren Faktoren abhängen. Zum einen weisen die verschiedenen Sorten in Abhängigkeit der jeweiligen Schaleneigenschaften (Stärke und Struktur) eine unterschiedliche Schrumpfneigung auf. Sorten wie Braeburn, Golden Delicious oder Elstar neigen z.B. eher zu Schrumpfungserscheinungen als Sorten wie z.B. Jonagold. Zum anderen wird die Ausprägung der Kutikula (Wachsschicht) auf der Epidermis der Früchte, die als Sperre für Wasserdampf und somit als Schutz vor Wasserverlust dient, vom Reifegrad der Früchte beeinflusst. Bei unreifen Früchten ist diese Wachsschicht nicht voll ausgebildet und die transpirationshemmende Wirkung ist dadurch geringer, die Früchte schrumpfen schneller. Generell transpirieren kleinere Früchte stärker als größere, bedingt durch das größere Verhältnis von Oberfläche und Volumen.

\section{MAßNAHMEN}

Eine zusätzliche Befeuchtung der Räume bringt nur bedingt Vorteile, da bei zu starker Entfeuchtung am Verdampfer, z.B. auf Grund schlechter Abstimmung der Anlage, auch das zusätzlich zugeführte Wasser wieder auskondensieren würde. Vielmehr steigt dadurch die Gefahr einer Vereisung der Verdampfer. Eine Befeuchtung von Holzkisten oder des Bodens vor Einlagerung kann jedoch deren Wasseraufnahme und damit die Transpirationsverluste der Früchte reduzieren.

Entscheidend für den Wasserverlust ist vor allem die optimale Abstimmung und Einstellung der Kälteanlage. Dabei ist zunächst beim Bau bzw. der Konzipierung der Anlage auf eine ausreichende Verdampferoberfläche zu achten. Liegen die Ursachen für eine zu hohe Entfeuchtung bereits in der Dimensionierung der Kälteanlage, so lässt sich dieser nur schwer entgegenwirken. Als Richtwerte für die Dimensionierung der Anlage gelten eine Kälteleistung von ca. 180 Watt sowie eine Verdampfer-Oberfläche von ca. 2 $\mathrm{m}^{2} /$ Tonne Apfel. Ist die Dimensionierung, wie häufig in alten Räumen, zu gering ausgelegt, kann manchmal nur ein Wechsel der Kältetechnik die Lösung sein. Ein zweiter, einfach zu beeinflussender Faktor ist die Einstellung der Maximal- und Minimalwerte bei der Temperatursteuerung $(\Delta \mathrm{T})$. Als Richtwert kann z.B. eine Hysterese von ca. $+/-0,3{ }^{\circ} \mathrm{C}$ in der Steuerung eingegeben

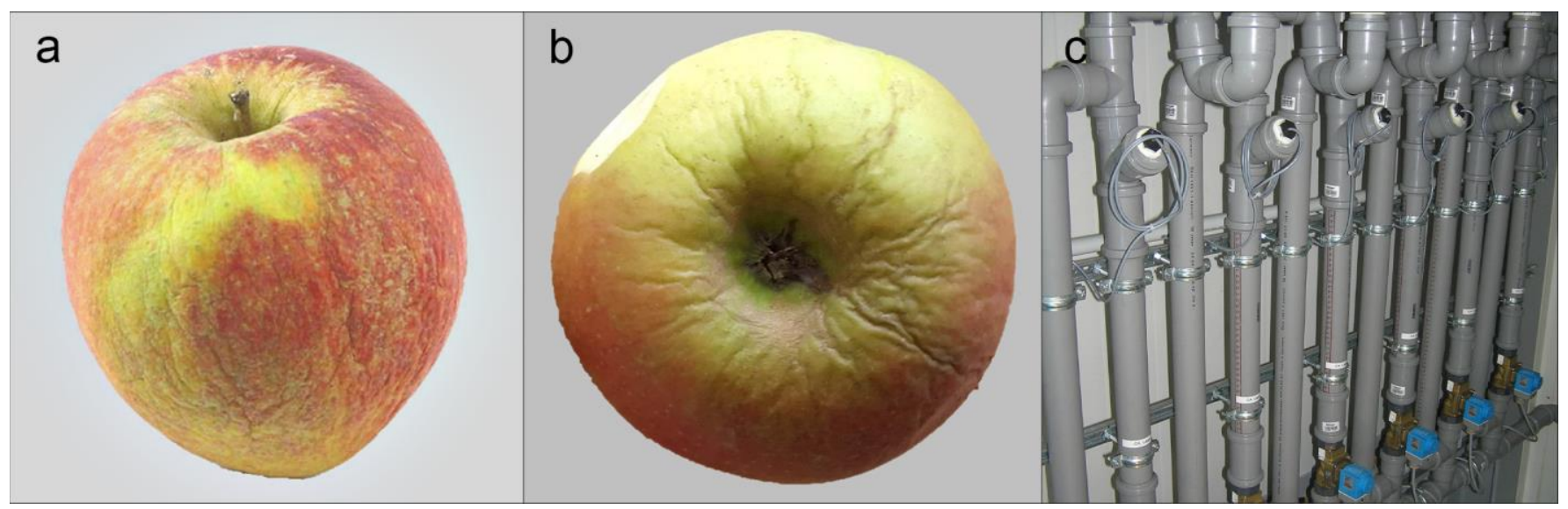


werden. Neben der Einstellung der Anlage sollte die Raumgröße an das Lagervolumen angepasst werden. Eine zu lange Lagerung in Räumen mit großem Leervolumen (z.B. Umschlagslager) ist nicht zu empfehlen. Zudem sollten vor Beginn der Lagersaison in den leeren Räumen alle Verdampfer bzw. Ventilatoren auf Funktionsfähigkeit geprüft und bei Bedarf ausgewechselt bzw. repariert werden. Ein erhöhter Wärmeeintrag in den Raum, z.B. durch schlecht isolierte Türen oder zu dünne Sandwichpaneele an den Wänden ist in der Regel nur schwer zu beheben. Hier muss geprüft werden, ob eine nachträgliche Isolierung der Räume möglich ist. Beim Befüllen der Räume im Herbst sollte auf ausreichende Stapelabstände geachtet werden, um das Abführen der Wärme zum Verdampfer zu ermöglichen und dadurch die Bildung von Wärmenestern und damit verbunden eine erhöhte Entfeuchtung der Ware zu vermeiden. Als grobe Faustzahl gilt ein Abstand von ca. $10 \mathrm{~cm}$ zwischen den Kisten, $50 \mathrm{~cm}$ auf der Druckseite, $40 \mathrm{~cm}$ auf der Ansaugseite, $20 \mathrm{~cm}$ seitlich zu den Wänden sowie $50 \mathrm{~cm}$ zur Decke. Markierungen auf dem Boden können dabei das optimale Einstapeln erleichtern.

Eine gute und technisch einfache Möglichkeit, um die tatsächliche Entfeuchtung der Früchte im Lager zu kontrollieren, ist die Installation einer Anlage zur regelmäßigen Erfassung der Kondenswassermengen (Abb. 4 c). Dabei wird das am Verdampfer kondensierte Wasser aufgefangen und mengenmäßig erfasst. Je nach Sorte sollte dies z.B. bei 3-4 Litern pro Tonne und Monat für die Sorten Cox Orange, Boskoop und Elstar, 2-3 Liter pro Tonne und Monat für Golden und Braeburn oder 1,5-2,5 Liter pro Tonne und Monat für Jonagold, Fuji und Idared liegen. Wird eine deutlich höhere oder niedrigere (z.B. bei Vereisen der Verdampfer) Abtauwassermenge gemessen, gilt es nach den Ursachen für die zu hohe bzw. zu niedrige Entfeuchtung zu suchen und entsprechende Maßnahmen zu ergreifen.

\section{SCHALENFLECKEN AN ELSTAR [25] [26] [27] [28] [29] [30]}

\section{SYMPTOME}

Schalenflecken werden gewöhnlich erst nach der CA/ULO-Lagerung sichtbar. Sie äußern sich durch kleine, leicht eingesunkene, bräunlich-schwarze Flecken, meist auf der grünen Grundseite der Frucht (Abb. 5 a).
Bei leichtem Befall sind meistens zuerst im Stielbereich und auf der Schulter der Frucht Symptome zu erkennen, häufig direkt anschließend an vorhandenen Berostungen. Mit zunehmendem Befall ist immer mehr der grünen Fruchtoberfläche betroffen. Teilweise sieht es aus, als würden die Schalenflecken vom Stiel zum Kelch hinunterfließen. Nur bei extrem starkem Befall findet man auch Schalenflecken im roten Deckfarbenbereich. Betroffene Schalenbereiche neigen zum Schrumpeligwerden (Abb. 5 b, c, d, e, f, $g, h)$.

\section{URSACHE}

Primäre Ursache für Schalenflecken sind Mikrorisse in der Fruchthaut, die durch längere Nässeperioden während der späten Fruchtentwicklung am Baum verursacht wurden. Während der Lagerung unter kontrollierter Atmosphäre sterben dann Zellen im Bereich dieser Mikrorisse ab und das Gewebe verbräunt.

\section{PHYSIOLOGISCHER HINTERGRUND}

Wie oben beschrieben sind nach bisherigem Kenntnisstand Mikrorisse die primäre Ursache für Schalenflecken. Treten diese Mikrorisse früh auf, also noch während der Zellteilungsperiode (bis ca. sechs Wochen nach der Blüte), verkorken diese und werden noch am Baum als Berostungen auf der Fruchtschale sichtbar. Später auftretende Mikrorisse können von der Frucht nicht mehr verschlossen werden und werden später als Schalenflecken sichtbar. Je häufiger, je länger und je später diese Feuchteperioden stattfinden, desto stärker der spätere Schalenflecken-Befall. Zusätzlich sind vor allem Früchte betroffen, die schlecht abtrocknen, z. B. Früchte aus dem Inneren von dichten sehr wüchsigen Bäumen an windgeschützten Standorten.

Schalenflecken treten nur nach CA-Lagerung und erst nach mindestens acht-wöchiger Lagerung auf. Je höher der Sauerstoffgehalt im Lager ist, desto stärker der spätere Befall. Beides, Dauer und Höhe der Sauerstoffkonzentration, spricht für einen Oxidationsprozess als Ursache für den einsetzenden Verbräunungsprozess. Warum Schalenflecken nicht bei unter Normalatmosphäre gelagerten Früchten auftreten, ist unklar. Auch ist nicht klar, warum Ethylen im Lager das Auftreten hemmt und eine 1-Metylcyclopropen-Behandlung dieses fördert. Die rissbedingte erhöhte Transpiration und der damit verbundene erhöhte Wasserverlust an den betroffenen Stellen verursachen mit großer Wahrscheinlichkeit das Einsinken der Flecken bzw. das regional verstärkte Schrumpeligwerden der Schale.

\section{VORBEUGUNG UND MAßNAHMEN}

Grundsätzlich sind bei Neupflanzungen möglichst regenarme und luftige Standorte auszuwählen. Da das Phänomen fast ausschließlich im Bereich der grünen Grundseite auftritt, sollte man bei Neupflanzungen farbige Mutanten bevorzugen.

Um das Abtrocknen der Früchte zu fördern, sollten alle Kulturmaßnahmen (insbesondere Ausdünnung und Wachstumsregulierung) auf die Erziehung eines ruhigen und gut belichteten Baumes abzielen. Farblose und kleine Früchte im Inneren des Baumes sind mit Hilfe einer gezielten Handausdünnung zu entfernen. Auf den Einsatz von hygroskopischen, also wasseranziehenden, Düngern oder Pflanzenschutzmitteln wie Kalziumchlorid sollte, wenn möglich, verzichtet werden. Einige Wochen vor der Ernte sind die Früchte mit einem optimalen Belichtungsschnitt konsequent freizustellen. Bei angekündigten langandauernden Regenereignissen zur Ernte sollte unter Berücksichtigung der fruchtphysiologischen Reife idealerweise vorher geerntet werden. Ein mehrmaliges Durchpflücken ist anzustreben.

Anfällige Früchte sollten nur unter Normalatmosphäre und zur besseren Qualitätserhaltung so kalt wie fruchtphysiologisch möglich gelagert werden (ideal: Stufenkühlung auf $-0,5^{\circ} \mathrm{C}$ ). Zusätzlich kann eine 1-Metylcyclopropen-Behandlung helfen, die Lagerungsdauer im einfachen Kühlhaus zu verlängern.

Für eine Langzeitlagerung unter CA/ULO-Bedingungen sollten möglichst nur unanfällige Früchte ausgewählt werden. Durch eine mindestens drei-wöchige Vorlagerung unter Normalatmosphäre kann das Schalenflecken-Risiko deutlich reduziert werden, sie verkürzt aber durch den Festigkeitsverlust auch die Gesamtlagerdauer. Die O2-Konzentration ist entsprechend des DCA-Prinzips so niedrig wie fruchtphysiologisch möglich einzustellen. $\mathrm{CO} 2$ hemmt mit zunehmender Konzentration das Auftreten von Schalenflecken, kann aber auch andere physiologische Störungen wie Schalennekrosen oder Fleischbräune fördern. Die Konzentration ist deshalb entsprechend der regionalen Empfehlung einzustellen. Auch Ethylen hat eine mindernde Wirkung, fördert aber 

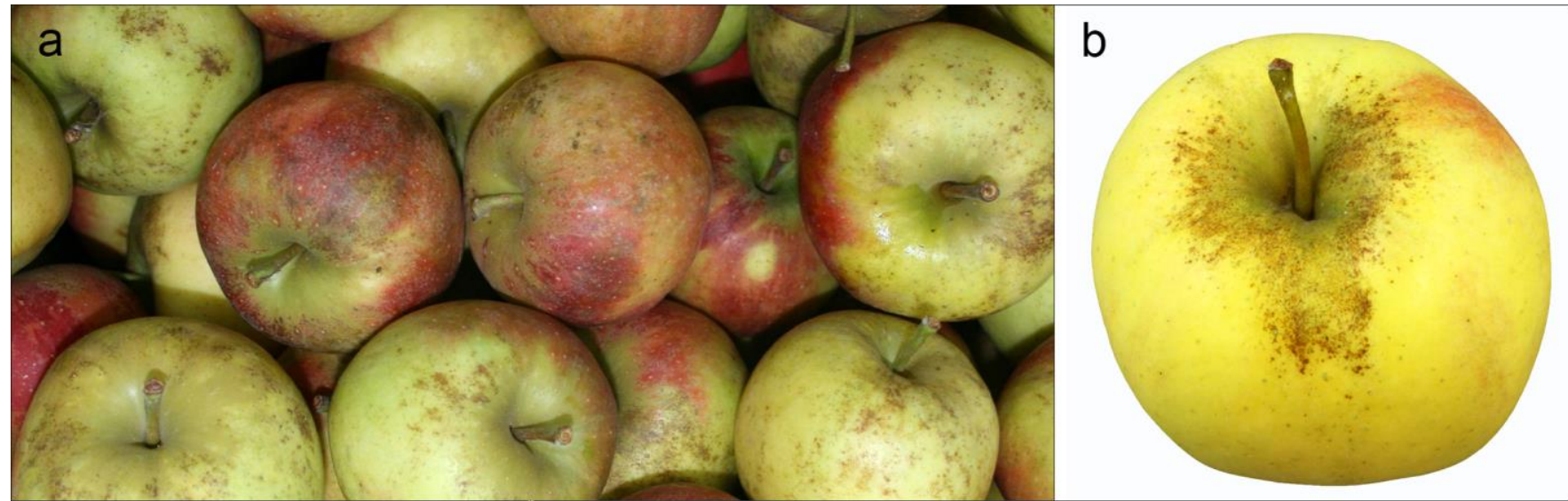

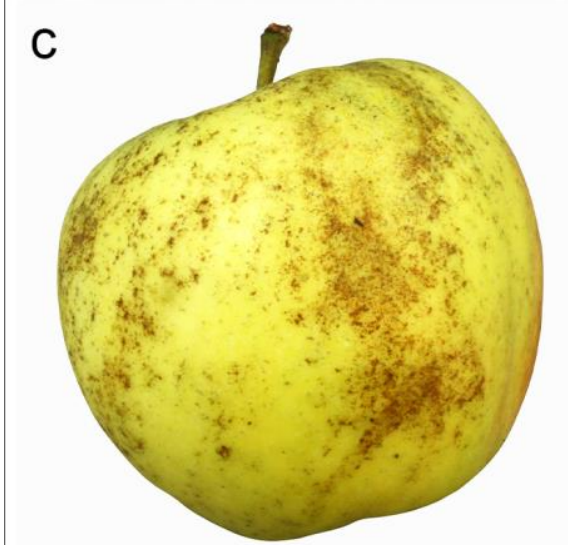

d

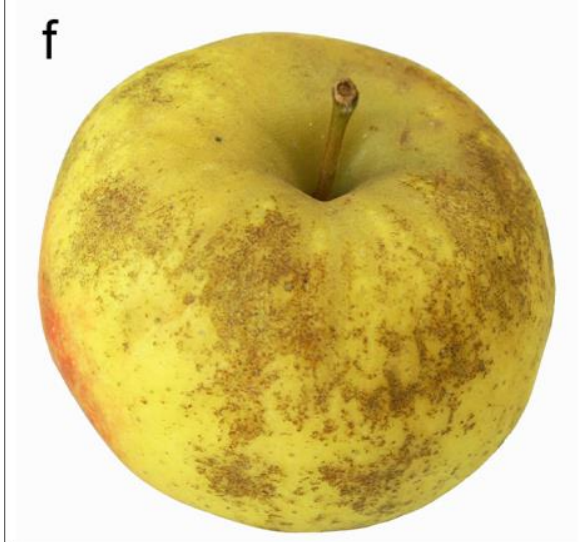

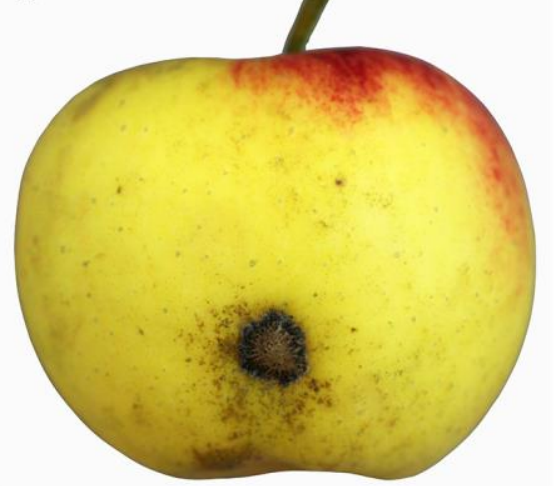

\section{g}

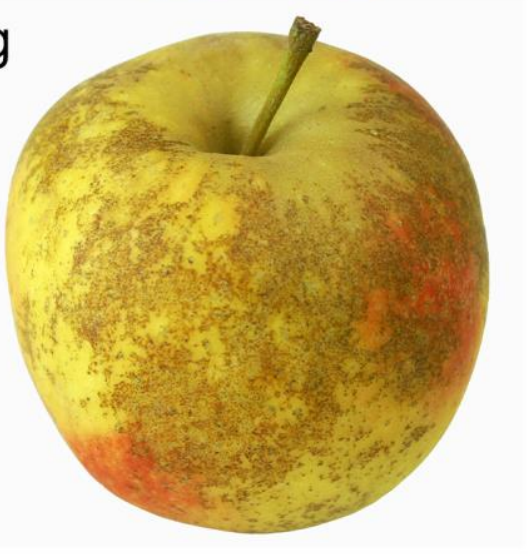

e
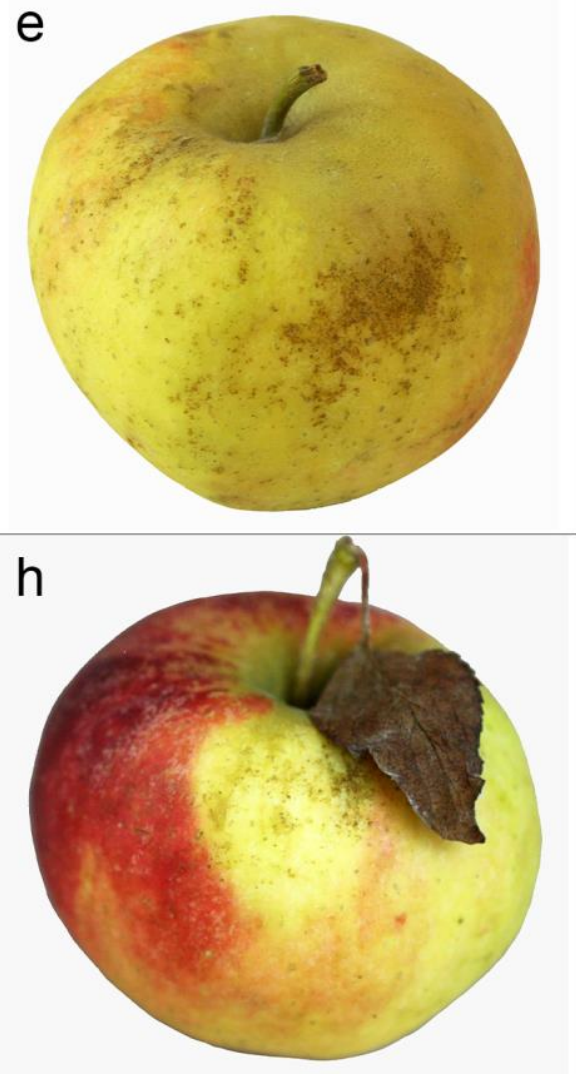

Abb. 5: Schalenflecken // Skin spots.

auch den Festigkeitsabbau, was gegen eine künstliche Erhöhung spricht. 1-Metylcyclopropen fördert den Befall unter CA/ULO-Bedingungen deutlich und sollte nur bei weniger anfälliger Ware und in Kombination mit den oben genannten Minderungsmaßnahmen eingesetzt werden.

Zur Beobachtung der Befallsentwicklung im CA-Lager ist es empfehlenswert, anfällige Früchte am Lagerfenster zu postieren, um mit Hilfe von regelmäßigen Fruchtprobenentnahmen einen beginnenden Befall rechtzeitig zu erkennen. Nach frühestens acht
Wochen CA-Lagerung wird ein Befall sichtbar und steigt danach deutlich an.

Andere Bezeichnungen: Schalenfleckchen, Skin spots (GB), Schilvlekjes (NL).

Anfällige Sorte: Elstar.
DRUCKSTELLEN - EIN SCHADEN, DER UNTER DIE HAUT GEHT [31] [32] [33] [34] [35] [36]

\section{SCHADBILD}

Äußere Qualitätsmängel des Apfels, wie z.B. Druckstellen, stellen ein weit verbreitetes Problem im Anbau und vor allem für die Vermarktung dar, da sie die Marktqualität und den Marktwert der Apfelfrucht erheblich mindern. Vor allem in den ersten Monaten der Lagerung von Äpfeln wird von der Praxis immer wieder von Qualitätseinbußen auf 


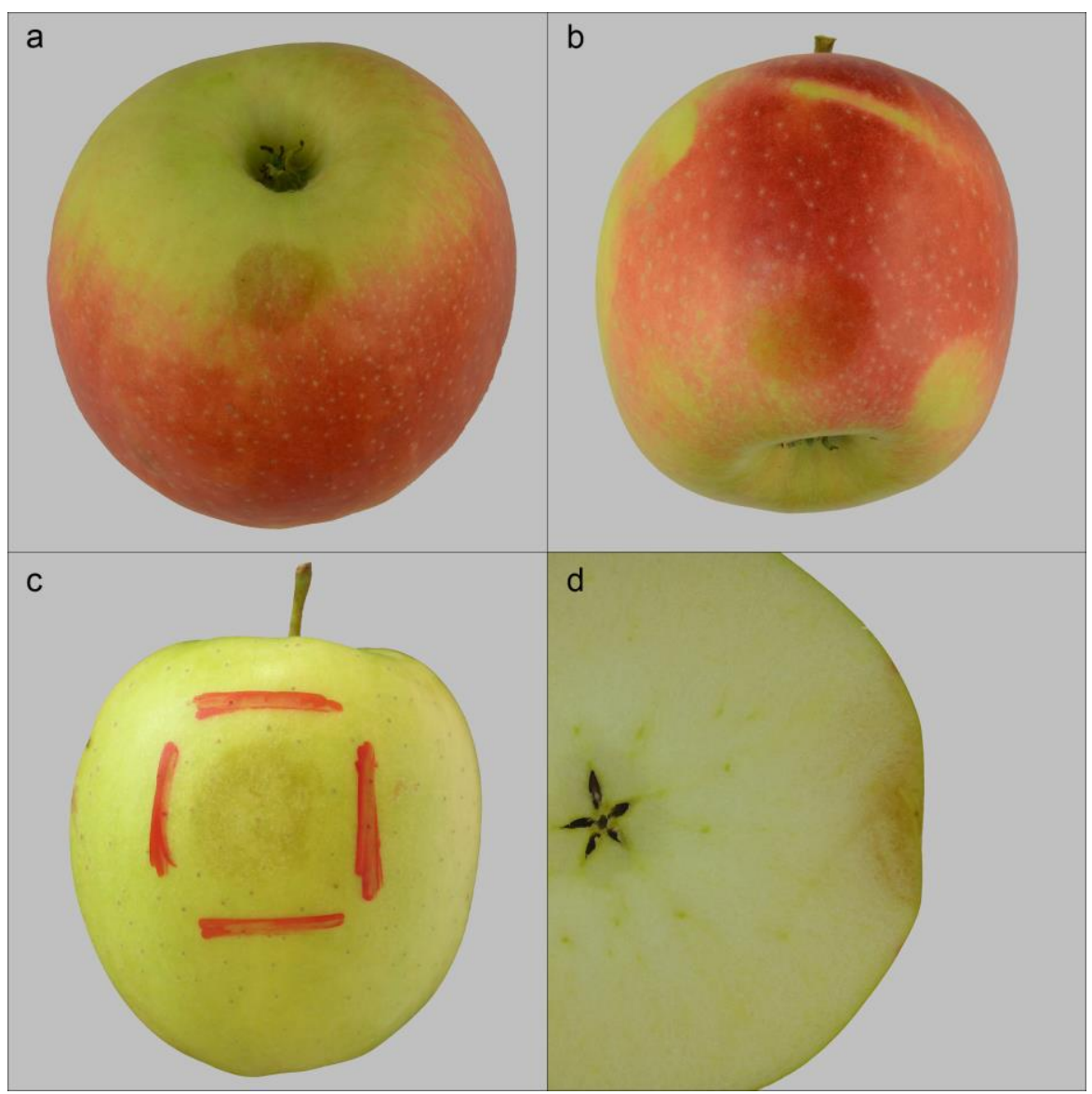

Abb. 6: Druckstellen // Bruising.

den Märkten berichtet, welche von mechanisch verursachten Schäden, wie eben Druckstellen, herrühren. Im Folgenden soll Aufschluss über die Symptome, die möglichen Ursachen und den physiologischen Hintergrund gegeben werden, um schließlich mögliche Maßnahmen der Vorbeugung von Druckstellen am Apfel vorzuschlagen.

\section{SYMPTOME}

Druckstellen äußern sich als kreisförmige bis elliptische Vertiefungen an der Oberfläche der Frucht (Abb. 6 a, b, c) und sind die häufigste Ursache für Oberflächenschäden bis hin zu tieferen Gewebeveränderungen (Abb. 6 d). Häufig weisen Druckstellen unmittelbar nach deren Verursachung keine Farbveränderung auf, sondern erst zu einem späteren Zeitpunkt, nach einigen Stunden. Vor allem zur Ernte und in den ersten Monaten der Lagerung treten Druckstellen häufig auf. Obwohl alle Sorten mehr oder weniger zu Druckstellen neigen, gibt es sortenspezifische Unterschiede in der Anfälligkeit: Als besonders anfällige Sorten gelten Golden Delicious und Cripps Pink/Pink Lady ${ }^{\circledR}$.

\section{URSACHE}

Druckstellen entstehen durch unsachgemäße statische oder dynamische Druckbelastungen der Früchte, beispielsweise durch einen konstanten Gewichtsdruck oder durch Reibungen und Stöße, während der Ernte, Anlieferung, Aufbereitung oder Vermarktung. Dabei kommt es nicht zu einem Aufbrechen der Fruchtschale, sondern zu einer Deformierung der Schale und des Fruchtfleisches mit einer Verschiebung und Beschädigung der darunterliegenden Zellschichten.

Es ist anzunehmen, dass Produktionsverhältnisse, die zu schweren Früchten mit großzelligem Gewebe und zu einer unausgewogenen Mineralstoffversorgung der Früchte führen, eine höhere Empfindlichkeit für Druckstellen verursachen.

\section{PHYSIOLOGISCHER HINTERGRUND}

Der aktuelle Kenntnisstand lässt vermuten, dass der zur Ernte in den pflanzlichen Zellen vorherrschende hohe Turgor (Druck des Zellsaftes auf die Zellwand der pflanzlichen Zellen) zu einem zerbrechlichen Gewebezustand der Frucht führt.
Durch unsachgemäße mechanische Belastung der Früchte zur Ernte kann es sehr leicht zu einer plastischen Deformierung der Oberfläche, mit einem Verlust der Struktur von darunterliegenden Zellschichten kommen. In schwerwiegenden Fällen können die Parenchymzellen (große Zellen unter den dichteren Zellschichten der Schale) zerstört werden. Als Folgereaktion schließt sich häufig die Verbräunung der Fruchtschale und des Fruchtfleisches an (Oxidation von Polyphenolen). Dieser Verbräunungs-Reaktion wirken im Apfel natürlich vorkommende Antioxidantien, wie das Vitamin C, entgegen. Daher kann sich der Druckfleck an einer Druckstelle später zurückbilden.

Während der Lagerung, mit laufendem Verlust des Wassergehalts der Äpfel, nimmt der Turgor in den Zellen ab und führt so zu einer schwächeren Empfindlichkeit für Druckstellen. Die Empfindlichkeit ist zur Ernte und bei tiefen Temperaturen am höchsten, nach der längerfristigen Auslagerung deutlich geringer.

\section{VORBEUGUNG UND MAßNAHMEN}

Das Pflückpersonal sollte fachmännisch geschult werden, mit welcher Technik die Früchte anzufassen und vom Baum zu trennen und wie sie ordnungsgemäß in die Großkisten zu entleeren sind. Bei druckempfindlichen Sorten sollte ein Erntebeginn am frühen Morgen und bei Nässe und Regen unterlassen werden. Weiters ist bei empfindlichen Sorten auch auf einen sorgsamen Umgang während der Sortierung und des Transports zu achten. Um Druckstellen, welche durch die Sortierung entstehen könnten vorzubeugen, empfiehlt es sich, die Kühlzellen einige Zeit vor der Sortierung zu öffnen und zu belüften. Dadurch kann der in den pflanzlichen Zellen vorherrschende Turgor gesenkt werden. Diese Maßnahme ist jedoch nur bei einer frühen Vermarktung der Früchte notwendig und ratsam.

\section{WEICHE SCHALENBRÄUNE [37]} [38] [39] [40] [41]

\section{SYMPTOME}

Weiche Schalenbräune äußert sich durch eingesunkene, unregelmäßig und bandförmig geformte, helle bis sehr dunkle Verbräunungen der Fruchtoberfläche. Die Krankheit ist nicht auf die Epidermis begrenzt, sondern greift auch im fortschreitenden Stadium auf das darunterliegende Gewebe über. Der betroffene Bereich weist eine glatte, oft straff 


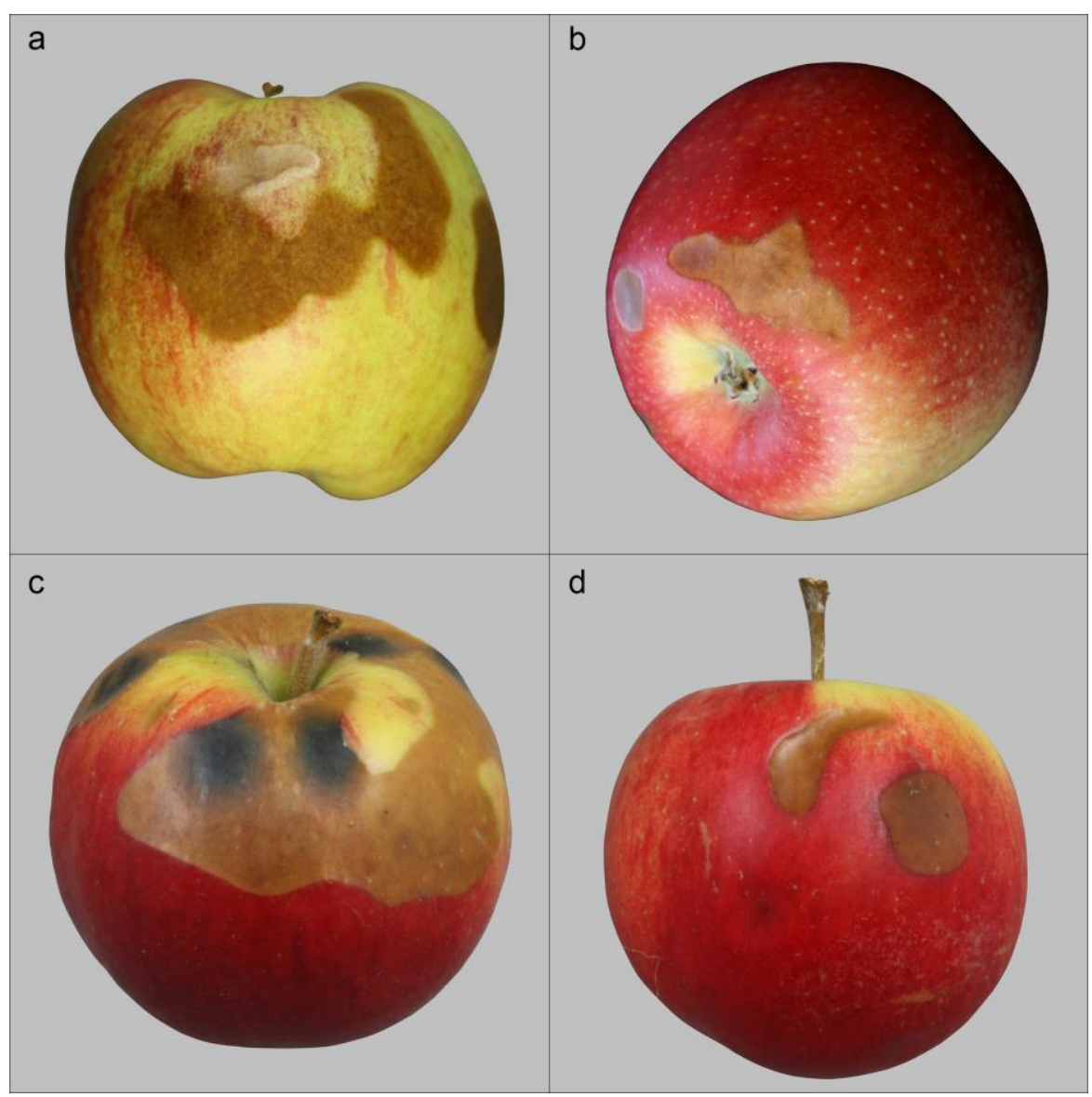

Abb. 7: Weiche Schalenbräune // Soft scald

gespannte Oberfläche auf, die sich samtweich anfühlt. Häufig kommt es zu Sekundärinfektionen durch Schwächepilze, die sich durch schwarze Flecken auf den verbräunten Schalenbereichen zeigen. Zusätzlich ist die Haut leicht einzudrücken und wird häufig durch Wundfäulepilze besiedelt. Der Schaden tritt sowohl im einfachen Kühlhaus als auch unter CA/ULO-Bedingungen auf.

\section{URSACHE}

Es handelt sich um keine Schalenbräune im eigentlichen Sinne. Ursache ist eine für die Sorte zu tiefe Lagertemperatur, bzw. ein zu schnelles Abkühlen der Früchte nach der Ernte.

\section{PHYSIOLOGISCHER HINTERGRUND}

Durch die zu kalte Lagerung bzw. zu schnelle Abkühlung noch sehr atmungsaktiver Früchte kommt es zu Stoffwechselstörungen und damit zur Erkrankung und letztlich zum Absterben der Zellen im betroffenen Schalenbereich. Parallel werden verstärkt Hexanol und dessen Ester gebildet. Je nach Sorte kann die Krankheit schon bei Temperaturen deutlich über $0{ }^{\circ} \mathrm{C}$ auftreten.

\section{FEHLENDER SCHALENGLANZ - TRÜBSCHALIGKEIT ODER WEI- ßER HAUCH? [42] [43] [44] [45]}

\section{SYMPTOME}

Als Trübschaligkeit (physiologisch bedingt) bezeichnet man Lufteinschlüsse in der Fruchthaut. Sie werden durch die geänderte Lichtbrechung sichtbar und äußern sich durch eine milchige Verfärbung der Fruchthaut. In unmittelbarer Nähe der Lentizellen ist die Trübung nicht zu finden, wodurch diese stärker pigmentiert hervortreten (Abb. 8 a, b, c, d). Die Trübung kann nicht von der Oberfläche entfernt werden, ohne die Wachsschicht der Frucht zu verletzen. Während der Lagerung breitet sich die Veränderung jedoch nicht weiter aus.

Dies stellt auch das Unterscheidungsmerkmal zum weißen Hauch (parasitär verursacht) dar, welcher durch Abreiben relativ einfach zu entfernen ist. Weißer Hauch zeigt sich als feiner, mattgrauer bis weißlicher Belag, welcher durch das Myzel eines Brandpilzes der Spezies Tilletiopsis verursacht wird (Abb. 8 e, f).

\section{URSACHE}

Die Lufteinschlüsse der Trübschaligkeit entstehen während der ersten 30 bis 60 Tage nach Blühende. In diesem Zeitraum findet die Zellteilung statt. Durch eine physiologische Störung bzw. Reizung der Fruchtoberfläche kann Luft zwischen den Wänden der pigmentierten Hypodermiszellen (Unterhaut) eingeschlossen und später, meist erst kurz vor der Ernte als Trübung wahrgenommen werden. Die Epidermis (Oberhaut) bleibt dabei vollständig intakt. Bei einem sehr starken Reiz kann die Epidermis beschädigt werden. Dies führt dann zu einer vernarbten Fruchtschale, was sich als Berostung zeigt. Lufteinschlüsse, ähnlich jenen der Trübschaligkeit, können auch während der Berostungsreaktion entstehen. Aus diesem Grund wird vermutet, dass Trübschaligkeit und Berostung die gleichen Ursachen haben.

Weißer Hauch wird im Gegensatz dazu durch alle pilzfördernden Bedingungen begünstigt: Klimatische Einflüsse, wie Regenperioden bei milden Temperaturen im Spätherbst, unter Hagelschutznetzen auf Grund der erhöhten Feuchtigkeit und bei Früchten aus dem Inneren der Bäume oder in feuchten Lagen. Durch die hohe Luftfeuchtigkeit im Lager können die Symptome 


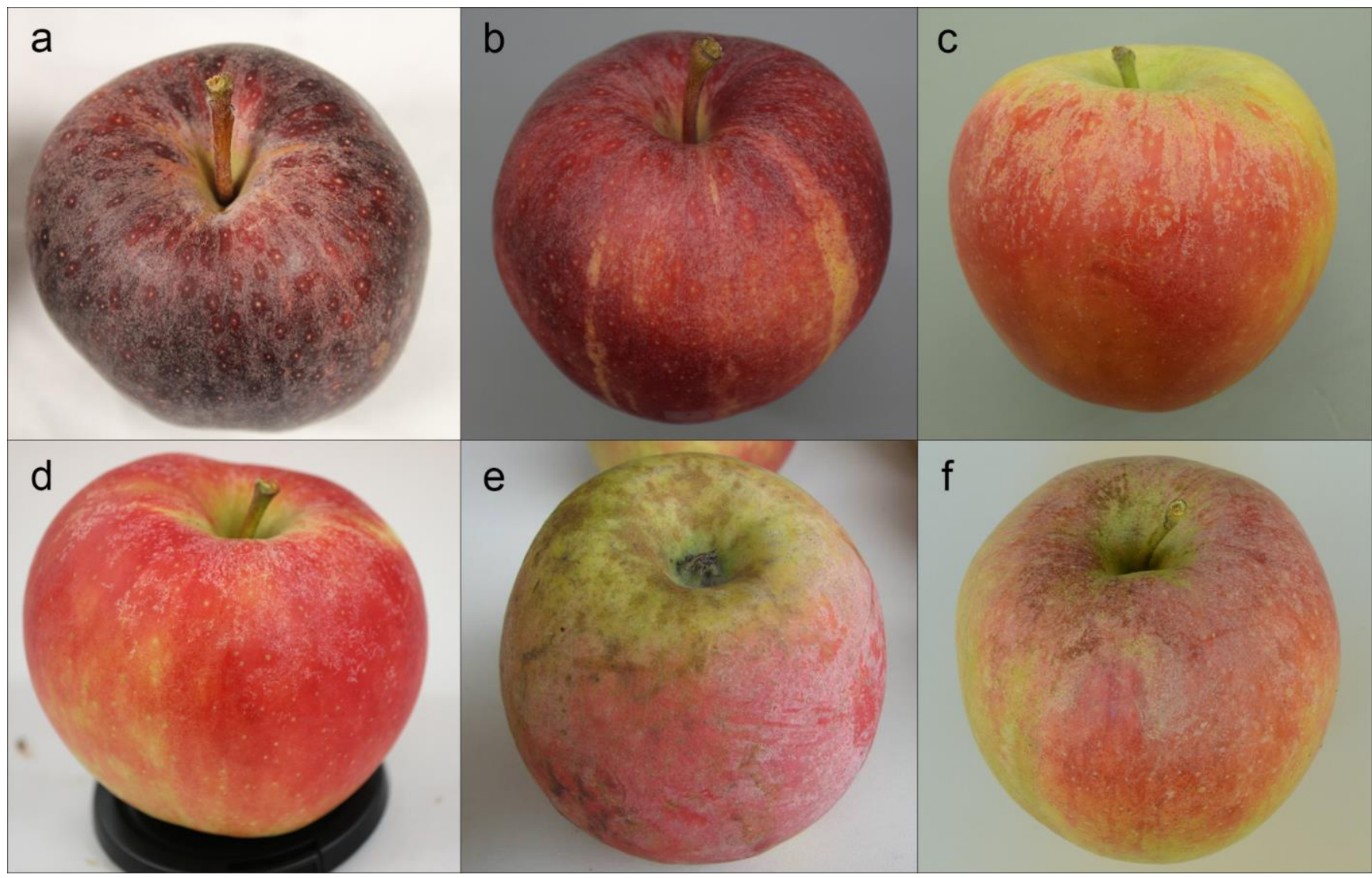

Abb. 8: Trübschaligkeit // Scarf skin.

im Kühl- oder ULO-Lager, bzw. in Feuchtekammern zunehmen. Nach Auslagerung können die Symptome durch Eintrocknung des Myzels verschwinden.

\section{VORBEUGUNG UND MAßNAHMEN}

Bei Trübschaligkeit wäre der Einsatz von Gibberellinen und/oder Regalis zur Vorbeugung theoretisch möglich, ist aber auf Grund der geringen wirtschaftlichen Bedeutung des Schadens nicht zu rechtfertigen. Bisher sind keine Vermarktungsprobleme durch Trübschaligkeit bekannt. Weitere Fragen zum Zusammenhang zwischen den bekannten Auslösern für Berostung, wie Feuchtigkeit oder Fungizid-Einsatz, und dem Auftreten von Trübschaligkeit müssen noch geklärt werden.

Bei weißem Hauch hat sich gezeigt, dass stickstoffhaltige Blattdünger die Ausprägung sichtbarer Symptome fördern, da der Stickstoff offenbar als Ernährung für die Pilze dient. Der Verzicht auf solcherlei Dünger, sowie gut durchlüftete Anlagen und Baumkronen, ebenso wie regelmäßige Unkrautbekämpfung können das Auftreten dieses Schadens eindämmen. Allerdings ist der entstehende wirtschaftliche Schaden als eher gering einzuschätzen. Weitere Untersuchungen zur Fungizid-Sensitivität können ausstehende Fragen zu Bekämpfungs- und Vorbeugungsstrategien beantworten.

Anfällige Sorten für Trübschaligkeit: Red Jonaprince, Golden Delicious, Gala, Elstar, Braeburn und andere.

Anfällige Sorten für Weißer Hauch: Topaz, Braeburn, Boskoop, Elstar, Jonagold und andere Sorten speziell Schattenfrüchte, tief am Baum hängende Früchte und spät geerntete Früchte.

\section{ALTERSFLEISCHBRÄUNE UND CA-BEDINGTE FLEISCHBRÄUNE AN GELAGERTEN ÄPFELN [46] [47] [48] [49] [50] [51]}

\section{SCHADBILD}

Unter dem Begriff Fleischbräune werden alle Erkrankungen des Fruchtfleisches zwischen Kernhaus und Schale bezeichnet, bei denen das Gewebe in Folge von Zellschädigungen verbräunt. Dabei kann das Auftreten von Fleischbräune durch verschiedene Faktoren verursacht werden. Dementspre- chend unterscheidet man in der Regel zwischen Altersfleischbräune, Kältefleischbräune und CA-bedingter Fleischbräune. Die Verbräunungen des Fruchtfleisches können je nach Ursache und Sorte unterschiedlich ausgeprägt sein. Im Folgenden werden die Alterfleischbräune sowie die CA-bedingte Fleischbräune näher betrachtet.

\section{SYMPTOME}

CA-bedingte Verbräunungen beginnen in der Regel im inneren Bereich des Fruchtfleisches, so dass bei anfänglichem Auftreten häufig eine gesunde Gewebeschicht zwischen der Verbräunung und der Fruchtschale zu beobachten ist (Abb. 9 a). Mit zunehmender Verbräunung breiten sich die Symptomstellen in Richtung Fruchtschale oder Kernhaus aus. Manchmal treten sekundär Kavernen, also kleine Hohlräume, im Fruchtfleisch auf (Abb. 9 b). Im Gegensatz dazu beginnt die Alters-Fleischbräune meistens im äußeren Bereich unmittelbar unter der Schale und breitet sich dann in das Fruchtinnere aus (Abb. 9 c). Die Verbräunungen sind bei der Altersfleischbräune nicht immer kreisförmig um die ganze Frucht angeordnet, sondern treten häufig anfangs nur partiell auf. Bei fortgeschrittener Alters- 


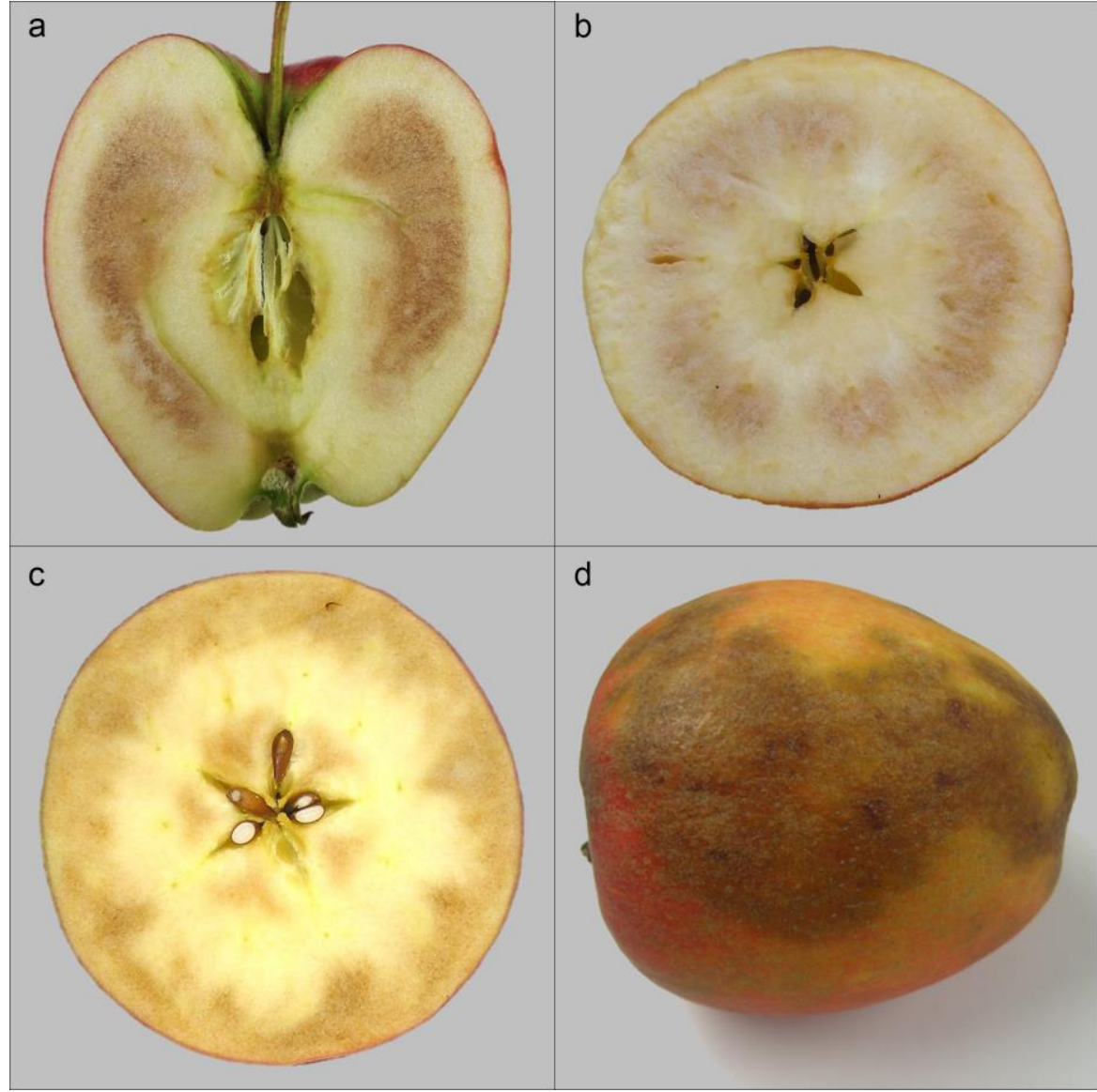

Abb. 9: Fleischbräune // Internal browning.

Fleischbräune können äußerlich dunkle, die Schale durchschimmernde Bereiche sichtbar werden (Abb. 9 d), meist verbunden mit einem massiven Weichwerden der Frucht.

\section{PHYSIOLOGISCHER HINTERGRUND UND URSACHEN DER CA-BEDING- TEN FLEISCHBRÄUNE}

CA-bedingte Fleischbräune wird durch Stoffwechselstörungen im Zuge der Fruchtatmung hervorgerufen. Zum einen wird bei der Fruchtatmung Energie frei, Voraussetzung für die Aufrechterhaltung lebenswichtiger Zellfunktionen der Frucht, wie z.B. dem Schutz vor Zellschädigungen. So kann es z.B. bei zu geringer Atmungsaktivität in Folge von Sauerstoffmangel zu Verbräunungen des Fruchtfleisches kommen (Gärschäden). Meistens jedoch wird die CA-bedingte Fleischbräune durch zu hohe CO2-Konzentrationen in der Atmosphäre verursacht. In der Regel erfolgt bei gut funktionierendem Gasaustausch, entsprechend dem Konzentrationsgefälle, ein Ausgleich der Sauerstoff

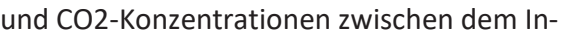
neren der Frucht und der Lageratmosphäre. Ist der CO2-Gehalt der Lagerluft zu hoch, verschiebt sich der Gradient zwischen Frucht und Lagerluft (CO2-Gehalt in der Lagerluft ist höher als in der Frucht) und das im Zuge der Atmung gebildete Kohlenstoffdioxid verbleibt länger im Fruchtfleisch. Bei der weiteren Atmung baut sich im Inneren der Frucht ein $\mathrm{CO} 2$-Überschuss auf, der zu Zellschädigungen und zur Braunfärbung der betroffenen Bereiche führt.

Zusätzlich wird der Gasaustausch von der sortenabhängigen Zellstruktur der Früchte beeinflusst. Untersuchungen haben gezeigt, dass festfleischige Sorten mit kompakteren Zellstrukturen, wie z.B. Braeburn, eine geringere Durchlässigkeit des Gewebes für Sauerhere Empfindlichkeit für CA-bedingte Fleischbräune aufweisen.

Vor allem während der Einlagerungsphase direkt nach der Ernte zeigen die Früchte eine höhere Atmungsaktivität als später während der Lagerung. Dadurch ist zu diesem Zeitpunkt die $\mathrm{CO} 2$-Produktion der Frucht deutlich höher. Häufig wird eine zu hohe CO2Konzentration in der Lagerluft durch mangelnde Belüftung bzw. CO2-Adsobtion während dieser Einlagerungsphase verursacht. stoff und $\mathrm{CO} 2$ und daher meistens eine hö-
Früchte von späteren Pflückterminen weisen auf Grund der fortgeschrittenen Reife meistens eine höhere Atmungsaktivität verbunden mit einer zunehmend geringeren Gasdurchlässigkeit des Gewebes auf und sind deshalb in der Regel empfindlicher für Fleischbräune als zum optimalen Termin geerntete Früchte.

\section{MAßNAHMEN}

Aus den genannten Gründen ist die Wahl des optimalen Erntetermins eine der wichtigsten Voraussetzungen zur Vermeidung von Fleischbräune, da vor allem zu spät gepflückte Partien eine erhöhte Empfindlichkeit für Fleischbräune aufweisen. Zu Problemen kann es häufig kommen, wenn die Früchte nach Einlagerung zwar zunächst unter Kühllagerbedingungen gefahren werden, auf Grund der hohen Dichtheit der Räume und der gleichzeitig hohen Atmugsaktivität der Äpfel sich die CO2-Konzentrationen im Lager jedoch innerhalb kurzer Zeit in einen für die Frucht schädlichen Bereich erhöhen. Deshalb ist von Anfang an auf ausreichende Lüftung oder CO2-Adsobtion zu achten. Bei manchen Sorten, wie z.B. Braeburn, hat sich eine ca. 3-wöchige Verzögerung bei der Einstellung der CA-Bedingungen als wichtige Maßnahme zur Reduzierung der Fleischbräune-Anfälligkeit bewährt. Eine CA-verzögerung ist jedoch nicht bei allen Fleischbräune anfälligen Sorten zu empfehlen. Bei der Sorte Santana z.B. haben Versuche eine Verstärkung der Fleischbräune durch CAVerzögerung gezeigt. Daher sind die sortenspezifischen Lagerempfehlungen immer zu beachten. $\mathrm{CO} 2$ empfindliche Sorten, wie z.B. Braeburn, Kanzi ${ }^{\circledR}$-Nicoter und Santana, sollten grundsätzlich bei möglichst niedrigen CO2 -Konzentrationen $(<1,2 \%)$ gelagert werden.

Wichtige Voraussetzung für eine zuverlässige CA-Steuerung ist zudem das regelmäßige Eichen der Messgeräte. Außerdem sollte zusätzlich mit einem Handmessgerät zweimal wöchentlich direkt am CA-Raum gemessen werden, um die tatsächlichen Konzentrationen im Raum zu prüfen.

\section{PHYSIOLOGISCHER HINTERRUND UND URSACHE DER ALTERS- FLEISCHBRÄUNE}

Bei der Alters-Fleischbräune handelt es sich um den Zusammenbruch des Gewebes infolge eines reifebedingten Zellwandabbaus und anschließenden Oxidationsprozessen im Fruchtfleisch. Fruchtatmung und Ethy- 


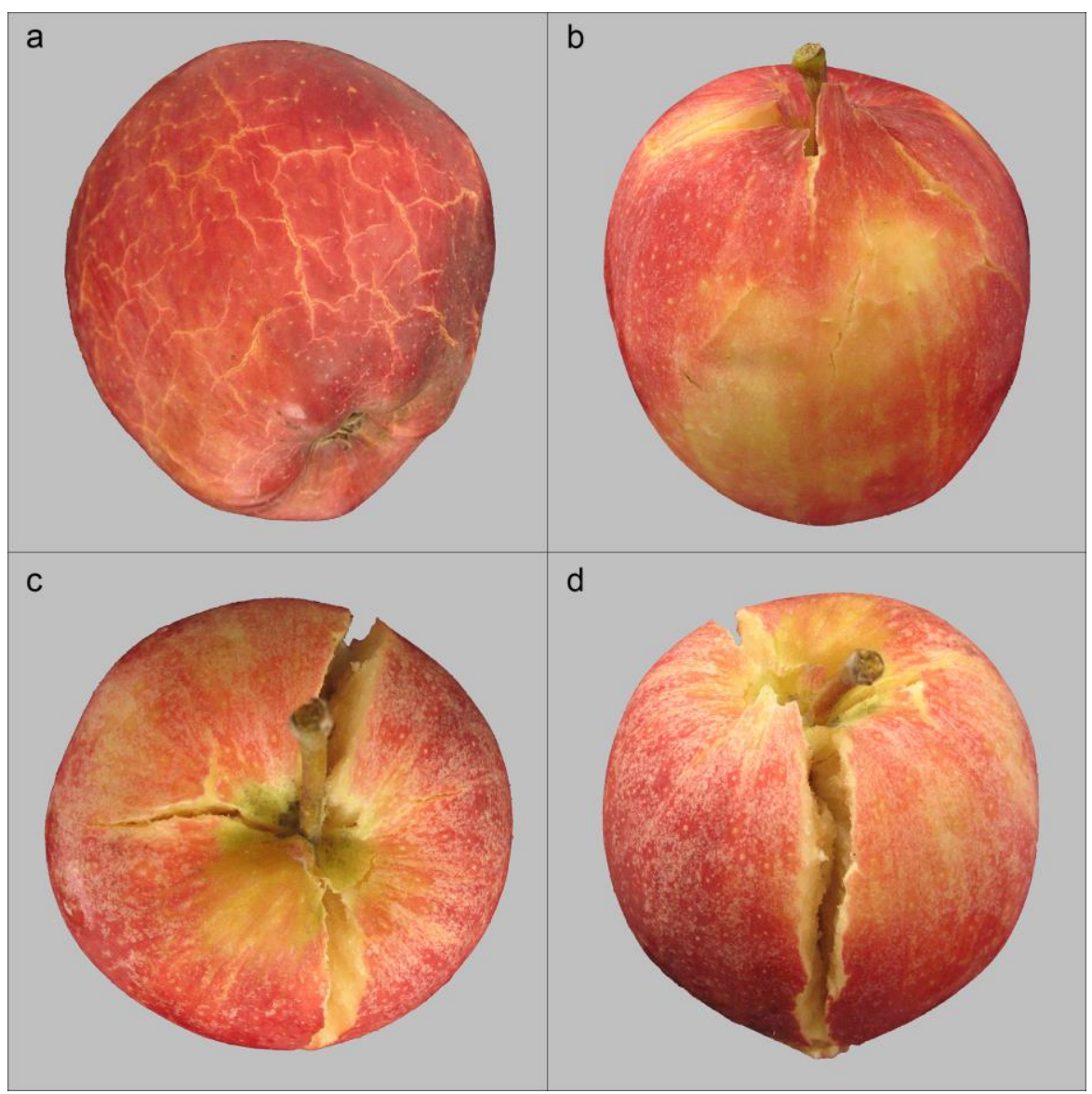

Abb. 10: Aufspringen // Mealy breakdown.

lenproduktion sowie die Beschaffenheit des Fruchtfleisches spielen hier eine wichtige Rolle. Spät geerntete Früchte weisen bereits zur Ernte einen fortgeschrittenen Zellwandabbau und damit beginnenden Festigkeitsverlust auf. Zudem erhöhen sich mit zunehmender Fruchtreife die Ethylenproduktion sowie die Atmung der Frucht. Beide Faktoren führen zu einer geringeren Haltbarkeit im Lager.

\section{MAßNAHMEN}

Alle Faktoren, welche zu einer verzögerten Fruchtreife beitragen, wirken dem Auftreten der Alters-Fleischbräune entgegen. Dazu zählt die sortenspezifische Lagerung bei niedrigen Temperaturen, unter kontrollierter Atmosphäre (CA, ULO), dynamisch kontrollierter Atmosphäre (DCA) oder die Applikation des Ethylenhemmstoffes 1-MCP. Bei zu reif geernteter Ware zeigen die genannten Lagerverfahren meist keine ausreichende Wirkung mehr. Der optimale Erntezeitpunkt muss je nach Anbauregion, Sorte, Lagerverfahren und Lagerdauer gewählt werden. Spät geerntete Früchte verhalten sich bei der Lagerung sehr instabil und
AUFSPRINGEN UND MEHLIGER ZERFALL AM APFEL WÄHREND DER LAGERUNG [52] [53] [54] [55] [56] [57]

\section{SCHADBILD}

Das Auftreten eines physiologischen Lagerschadens beim Apfel wird von verschiedenen Faktoren beeinflusst: So fördern, neben ungünstigen Wachstums- und Witterungsbedingungen, auch ein nicht idealer Erntezeitpunkt und ungeeignete Lagerbedingungen die Entwicklung eines Schadens und können schlussendlich zu dessen Auftreten während der Lagerung führen. Die Einhaltung des optimalen Erntefensters zur Erhaltung der Fruchtqualität spielt in diesem Zusammenhang eine bedeutende Rolle: Erfolgt die Ernte der Früchte beispielsweise verspätet, so kommt es während der Lagerung zu Überreife und Alterung der Früchte. Dies wiederum hat maßgeblichen Einfluss auf die Entwicklung der inneren Fruchtqualität: Die Festigkeit des Fruchtfleisches und der Gehalt an Säure in den Früchten nehmen mit zunehmender Reife ab und vermindern dadurch die Lagerfähigkeit. Zudem verlieren die Äpfel an Knackigkeit bzw. Saftigkeit, weil sie während der Lagerung nicht weiterreifen, sondern auf Grund der Überreife altern. Neben der Veränderung der inneren Qualität, fördert eine späte Ernte auch das Auftreten von äußerlich sichtbaren Schäden, wie beispielsweise Aufspringen. Vor allem übergroße und zu spät geerntete oder überlagerte Früchte sind bei zu hoher Luftfeuchtigkeit und Temperatur im Lager gefährdet für Aufplatzen.

\section{SYMPTOME}

Das Schadbild zeigt anfänglich kleine, oberflächliche Risse der Fruchthaut (Abb. 10 a, b), welche mit zunehmender Reife, bzw. Lagerdauer größer werden und vorwiegend, wenn auch nicht ausschließlich, im zentralen Bereich der Früchte lokalisiert sind. Meist reißt nicht nur die Haut, sondern auch ein Teil des darunterliegenden Fruchtfleisches auf (Abb. 10 c, d). Das Fruchtfleisch der aufgesprungenen Früchte weist eine mehlige, teils trockene Konsistenz auf, die Frucht verliert zunehmend an Saftigkeit und Geschmack. Je nach Sorte verändert sich die Farbe des Fruchtfleisches an den aufgeplatzten Stellen zu einem schmutzigen Cremeweiß bis Hellbraun. Häufig kommt es an den aufgesprungenen Stellen zu Infektionen 

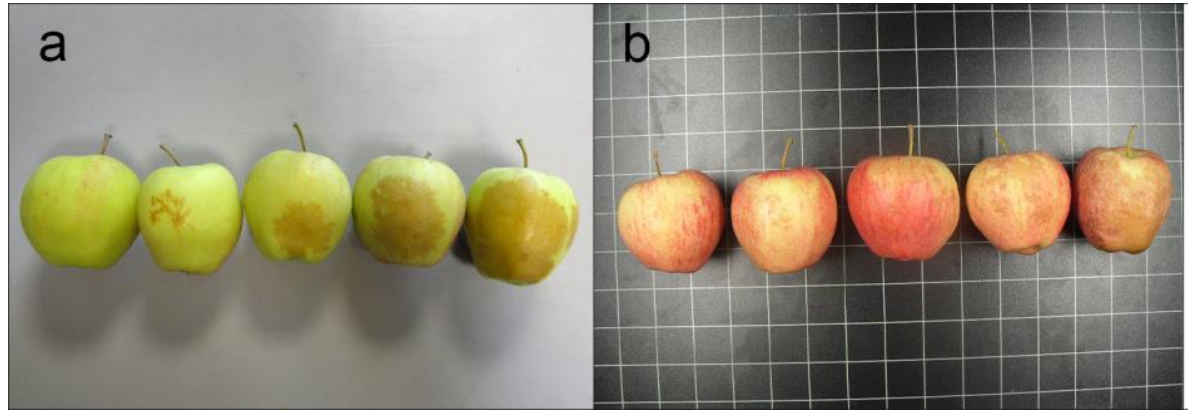

\section{C}

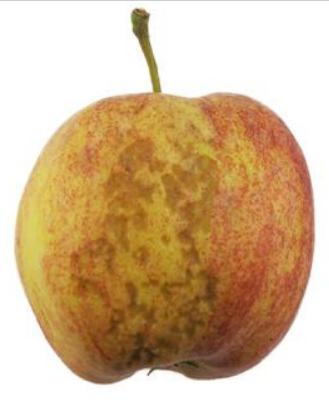

Abb. 11: Schalenverätzungen // Skin necrosis.

durch pilzliche Wundparasiten und die aufgeplatzten Stellen beginnen zu faulen.

\section{URSACHE}

Ein zu spätes Ernten, ein zu ausgedehnter Einlagerungsverzug oder ein zu langes Lagern der Früchte bzw. unvorteilhafte Lagerungsbedingungen gelten vor allem als die Ursachen des Schadens. Das Ausmaß der Symptome steigt bei einer Lagerung mit hoher Luftfeuchtigkeit und hoher Temperatur an.

\section{PHYSIOLOGISCHER HINTERGRUND}

Das Auftreten des Schadens ist eng mit der Alterung (Seneszenz) und der Lagerdauer der Früchte in Verbindung zu setzen. Studien deuten darauf hin, dass bei überreifen Früchten und zu langen Lagerzeiten die Kontakte zwischen den Zellen des Fruchtfleisches schwächer werden und es so zu einem alterungsbedingten Lockerwerden des Zellzusammenhaltes kommt. Dies ist vor allem auf Strukturveränderungen in den Mittellamellen (Bestandteile der pflanzlichen Zellwand) der Fruchtzellen zurückzuführen. Durch das altersbedingte Auflösen der Mittellamellen wird die Zellform immer kugeliger, der Raumbedarf der Zellen vergrößert sich und infolge auch der Druck auf die Hautschichten. Hinzu kommt, dass die Früchte bei der kommerziellen Apfel-Lagerung üblicherweise einer hohen relativen Luftfeuchte ausgesetzt sind und so der Turgor (Druck des Zellsaftes auf die Zellwand der pflanzlichen Zellen) erhalten bleibt. Der erhöhte Innendruck kann schlussendlich die sowieso durch den Reifungsvorgang alterungsgeschwächte Haut und das darunterliegende Fruchtfleisch zum Platzen bringen. Das Fruchtgewebe wird beim Kauen meist als mehlig empfunden, da die Zellen durch Biss nicht mehr aufplatzen und dadurch ein knackiges Frischegefühl erzeugen, sondern der Zellverbund sich bei Druck nur mehr verschiebt.

\section{VORBEUGUNG UND MAßNAHMEN}

Grundsätzlich kann Alters-Aufspringen durch eine termingerechte Ernte der Früchte bzw. durch die korrekte Einhaltung des empfohlenen Erntefensters, sowie ein rechtzeitiges Auslagern der Früchte und ideal angepasste Lagerungsbedingungen vermieden werden. Der optimale Bereich der relativen Luftfeuchte und der kontrollierten Atmosphäre, außerdem die ideale Lagertemperatur einer jeden Sorte sollte während der gesamten Lagerperiode eingehalten werden.

\section{WENN ES ZU SCHNELL GEHT - SCHALENVERÄTZUNGEN IM CA- LAGER [58] [59] [60] [61]}

\section{SCHADBILD}

Für einige Apfelsorten kann es großen Stress bedeuten, plötzlich vom Baum direkt ins CA Lager gebracht zu werden. Die Kohlendioxidgehalte sind einfach zu unterschiedlich und es bleibt keine Zeit zur Anpassung. Die Folgen sind sowohl außen auf der Schale als auch im Fruchtfleisch sichtbar. Im Folgenden wird auf die äußerlich sichtbare Schalenverätzung näher eingegangen.

\section{SYMPTOME}

Schalenverätzungen durch $\mathrm{CO} 2$ sind durch ein partielles Einsinken der Schale gekennzeichnet und treten meist auf der weniger ausgefärbten Seite der Frucht auf. Besonders betroffen sind unterentwickelte, schlecht gefärbte Äpfel (Schattenfrüchte, z.B. Cameo) (Abb. 11 a, b). Die betroffene Schalenfläche ist deutlich scharf abgegrenzt. Die Ränder verlaufen sehr unregelmäßig (Abb. 11 c). Die Symptome sind nicht unbedingt sofort nach dem Stress sichtbar. Sie können anfangs partiell punktförmig auftreten aber mit zunehmender Dauer der $\mathrm{CO} 2$
Exposition bzw. Höhe der Konzentration, zu großen Flächen wachsen. Die Fläche ist zu Anfang meist hellbraun und verfärbt sich im weiteren Verlauf dunkelbraun. Im Gegensatz zur gewöhnlichen Schalenbräune, nimmt die betroffene Schalenfläche nach beendeter $\mathrm{CO} 2$ Exposition nicht weiter zu.

\section{URSACHE}

Der Schaden tritt meist nur nach einer zu schnellen CA-Einstellung während der ersten vier bis sechs Wochen im Lager auf, wodurch bei sonst unempfindlichen Sorten das $\mathrm{CO} 2$ zu einem Problem werden kann. Hauptursache ist eine zu hohe $\mathrm{CO} 2$ Konzentration in der Lageratmosphäre. Das Auftreten des Schadens ist stark sortenabhängig. Auch eine hohe Atmungsintensität bei der Einlagerung spielt eine Rolle. Biochemische Ursachen sind weitestgehend unbekannt.

\section{VORBEUGUNG UND MAßNAHMEN}

Neben der Wahl des optimalen Erntetermines sollte bereits in der Anlage auf triebruhige, lichte Bäume geachtet werden. Bei gefährdeten Partien sollte die CA Einstellung erst vorgenommen werden, wenn sich der Metabolismus der Früchte angepasst hat. Dies bedeutet den $\mathrm{CO} 2$ Gehalt während der ersten zwei Monate bei maximal 1,5\% zu belassen und später auf Normalwerte zu erhöhen, bzw. die Früchte 3 Wochen lang im normalen Kühllager zu lagern. Bei Anwendung des Reifehemmstoffes 1-Methylcyclopropen (1-MCP) muss auf ein verzögertes Einstellen der CA Bedingungen besonders geachtet werden. Eine mögliche Ursache ist die Reifeverzögerung und der damit unterbundene Abbau der Sensitivität der Äpfel gegenüber $\mathrm{CO} 2$, welche durch Kühllagerung (hier: Frischluft) der Äpfel auftritt.

Anfällige Sorten: Golden Delicious, McIntosh, Empire, Cortland, Jonathan, Kanzi ${ }^{\circledR}$ Nicoter, Elstar, Gala, Fuji, Cameo, Braeburn, Topaz. 


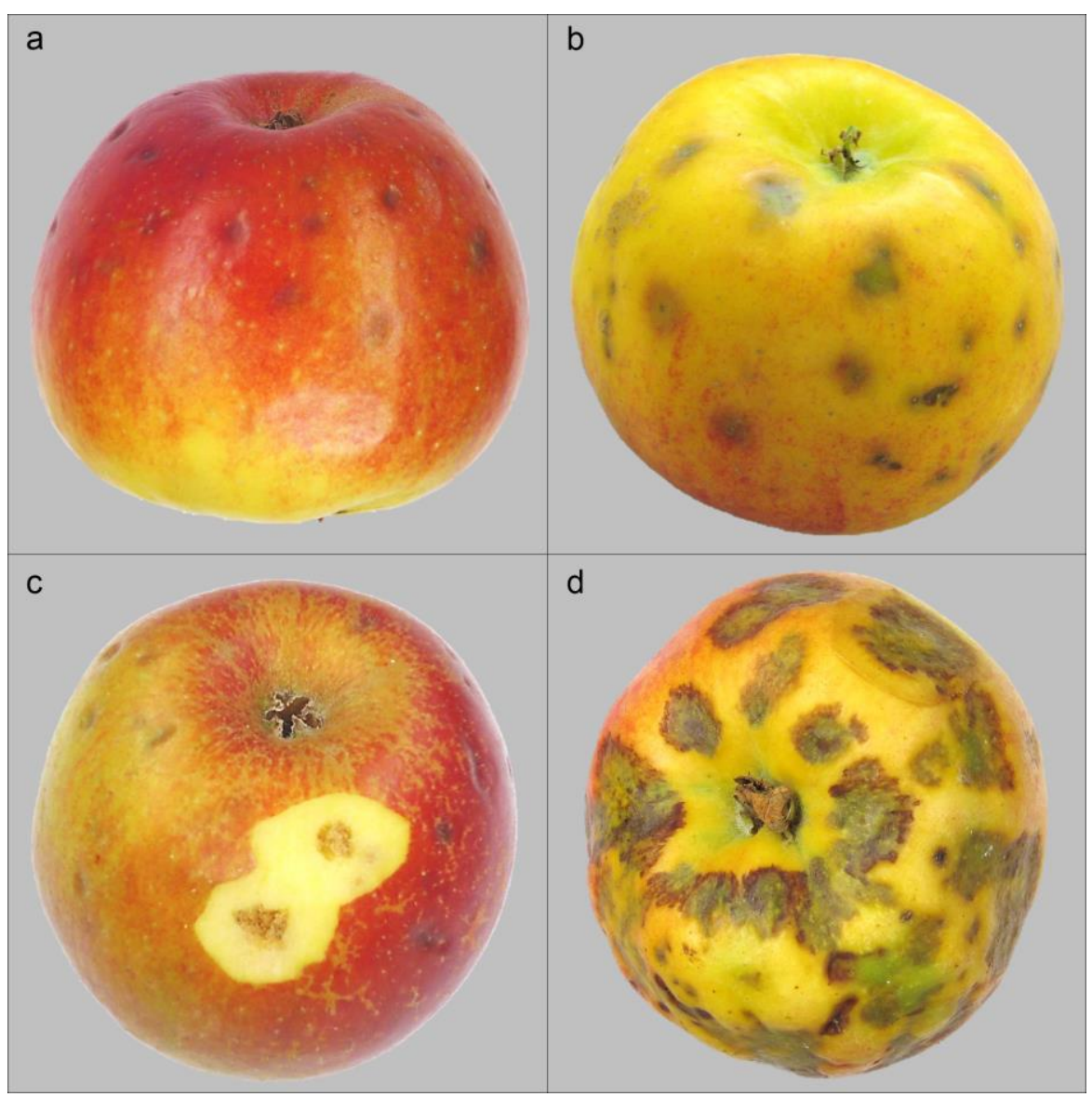

Abb. 12: Stippigkeit // Bitter pit.

\section{STIPPE AN GELAGERTEN ÄPFELN [62] [63] [64] [65]}

\section{SCHADBILD}

Auch wenn die Symptome physiologischer Lagerschäden meist erst während der Lagerung oder nach Auslagerung sichtbar werden, liegen die Ursachen häufig schon weit vor der Ernte. Hier können sowohl ungünstige Wachstums- und Witterungsbedingungen als auch Fehler bei der Durchführung von Kulturmaßnahmen (Schnitt, Behangsregulierung, Düngung usw.) mögliche Ursachen sein. In der Folge kann es häufig zu Stoffwechselstörungen (z.B. Glasigkeit) oder Mangelerscheinungen (z.B. Stippigkeit, Lentizellenflecken) in den Früchten kommen, welche sich negativ auf die Lagerfähigkeit auswirken und letztendlich zum Auftreten von sichtbaren Schäden führen können. Ein weit bekanntes physiologisches Problem, welches bereits vor der Ernte verursacht wird, ist das Auftreten von Stippigkeit.

\section{SYMPTOME}

Symptome der Stippigkeit treten meist erst im Lager oder sogar erst während der Nachlagerung (Vermarktung) auf, können jedoch bei massivem Mangel auch bereits am Baum zu sehen sein. Die Früchte zeigen dunkelgrün bis braun gefärbte, durchscheinende Flecken mit eingesunkener Schale (nekrotische Läsionen), meist beginnend im Bereich der Kelchgrube (Abb. 12 a, b). Das unter den Flecken liegende Gewebe ist verkorkt, jedoch meist nur oberflächlich direkt unter der Schale (Abb. 12 c). Bei massiver Stippigkeit können die Flecken auch flächiger auftreten (Abb. 12 d).

\section{PHYSIOLOGISCHER HINTERGRUND UND URSACHEN}

Verantwortlich für das Schadbild ist ein Calciummangel der Frucht. Die pflanzliche Zellwand besteht zu großen Teilen aus Pektin. Calcium hat die Eigenschaft, diese Pektinketten in der Zellwand, vor allem in den Mittellamellen, also dem Bereich, der die einzelnen Zellen miteinander verbindet, zu vernetzen. Dadurch wirkt Calcium als eine Art
Kittsubstanz im Gewebe. Bei Mangel an Calcium kommt es demzufolge zum Zusammenbruch der Zellstruktur und damit zum Auftreten von Stippe. Die Symptome beginnen meist im unteren Bereich der Frucht, nahe der Kelchgrube, da dort die Leitungsbahnen in der Frucht enden und dadurch ein Mangel an Calcium zuerst in diesem Bereich auftritt. Kalium und Magnesium können in der Zellwand "als Gegenspieler" zum Calcium wirken, d.h. eine Überversorgung mit einem der beiden Nährstoffe kann Calciummangel begünstigen und ein Ungleichgewicht in der Mineralstoffversorgung hervorrufen. Deshalb sollte tendenziell immer eher ein niedriges Verhältnis zwischen Kalium und Calcium angestrebt werden (Richtwert z.B. bei Sorte Cox Orange < 20).

Das Calciumangebot im Boden ist nur zum Teil für das Auftreten von Calciummangel von Bedeutung. Entscheidend ist vielmehr die Verteilung der Mineralstoffe in der Pflanze. Grundsätzlich wird Calcium über den Boden nur bis zum T-Stadium, also bis zum Ende der Zellteilungsphase, aufgenommen. Der Calciumtransport in der Pflanze erfolgt dabei im Xylem (Wasserleitungsbahnen) und ist daher besonders zu stärker transpirierenden Organen hingerichtet (Triebe, Blätter). Dies erklärt auch, warum Früchte von Bäumen mit starkem Triebwachstum bzw. schwachem Fruchtbehang zu einer verstärkten Stippeanfälligkeit neigen, da zu Gunsten der stark wachsenden Blätter und Triebe weniger Calcium in die Früchte eingelagert wird. Dementsprechend können alle Maßnahmen, welche zu einem verstärkten Triebwachstum führen, das Auftreten von Stippe fördern. Dazu zählen z.B. eine Überversorgung mit Stickstoff oder zu starke Schnittmaßnahmen im Winter. Nach dem T-Stadium beginnt die Frucht im Zuge der Zellstreckung an Kaliber zuzulegen. Da während dieser Phase keine Calciumaufnahme mehr über den Boden erfolgt, kommt es in den Früchten mit zunehmendem Fruchtkalieber zu einer Verdünnung der Calciumkonzentration. Deshalb neigen vor allem Früchte mit Übergrößen zu Stippigkeit. Bei hohen Temperaturen und Trockenheit nach der Blüte ist die Verteilung des Calciums durch den Saftstrom ebenfalls gestört und es soll sogar zum Rücktransport von den Früchten in die Blätter kommen. Grundsätzlich haben vor allem südlichere Anbauregionen mehr Probleme mit Stippe. 
VORBEUGUNG UND MAßNAHMEN

Zur Vorbeugung von Stippe oder anderen Stoffwechselstörungen ist auf ein ausgewogenes Verhältnis zwischen Triebwachstum und Fruchtbehang durch mäßigen Schnitt und angepasste Behangsregulierung zu achten. Eine Überversorgung der Bäume mit Stickstoff (fördert das Triebwachstum) sowie Kalium und Magnesium (wirken antagonistisch zum Calcium in der Zellwand) ist zu vermeiden.

Die Empfindlichkeit für Calciummangelsymptome ist stark sortenabhängig. Als empfindlich gelten z.B. Braeburn, Red Delicious, Golden Delicious, Jonagold, Granny Smith, Glockenapfel, Ontario oder Cox Orange. Hier empfiehlt es sich bei Problemen mit Calciummangel ab dem TStadium Ca-Spritzungen durchzuführen. Als effektive und kostengünstige Möglichkeit hat sich dabei die Anwendung von Calciumchlorid (0,5-0,8\%) bewährt. Zahlreiche Versuche haben gezeigt, dass eine nur ein- bis zweimalige Anwendung von $\mathrm{CaCl} 2$ vor der Ernte keine ausreichende Wirkung zeigt. Daher sollte ab Juli mindestens 6-8 Spritzungen durchgeführt werden. Die Calciumkonzentration in der Frucht kann dadurch um ca. $10-15 \%$ erhöht werden. Um mögliche Schäden an Blättern oder Früchten zu vermeiden und eine gute Aufnahme sicher zu stellen sollten die Calciumspritzungen möglichst an bedeckten, trockenen Tagen bzw. bei sonnigen, heißen Tagen nur in den kühleren Morgen- oder Abendstunden durchgeführt werden. Eine gleichmäßig gute Benetzung der Früchte ist zusätzlich eine wichtige Voraussetzung für eine gute Wirkung. Oft teure Calcium-Spezialdünger haben in Versuchen im Vergleich zu $\mathrm{CaCl} 2$ leider nicht immer die gewünschte Wirkung gezeigt.

Da das Risiko für Stippe und zahlreiche andere Lagerschäden mit zunehmender Fruchtreife steigt, sollte unbedingt auf den optimalen Erntetermin geachtet werden. Je ungünstiger die anschließenden Lagerbedingungen, umso schneller treten bei kritischen Partien Stippesymptome auf. Deshalb sollten die optimalen, sortenspezifischen Lagerbedingungen genutzt werden.

\section{ZUSAMMENFASSUNG}

Auch heute noch führen physiologische und parasitäre Schäden, welche sich während der Lagerung von Apfelfrüchten entwickeln können, zu erheblichen wirtschaftlichen Verlusten. Um den Lagerungsprozess von Äpfeln effizienter zu gestalten, wurde dank eines dreijährigen Interreg-V-EU-Projektes, ein Software-gestütztes Bestimmungssystem zur Reduzierung von Lagerschäden im Obstbau entwickelt: Frudistor, aus 'fruit disorders storage'. Diese Web-Applikation kann durch ihre einfache, praktische und kostenlose Anwendung - zugänglich unter http://www.frudistor.de von jeglichem digitalen Endgerät aus (PC, Tablet bzw. Smartphone) - Produzenten, Lagertechniker, Handelsorganisationen und Konsumenten dabei begleiten, die verschiedenen Schäden, die während der Lagerung von Früchten entstehen können, zu identifizieren und zu erkennen, um ihnen vorbeugen zu können und sie zu vermeiden. Dies wird mit Hilfe eines ausgeklügelten, einfachen Filtersystems realisiert. Die Software enthält mehr als 40 technische Datenblätter (derzeit auf Italienisch, Deutsch, Englisch und Niederländisch) und Bilder, die es ermöglichen, den Ursprung von Schäden schnell zu erkennen und somit eine Vermeidung zu gewährleisten. Der folgende Artikel will die Erkenntnisse dieses Projekts sammeln und stellt die wichtigsten Schäden vor, die während der Lagerung von Äpfeln auftreten können.

\section{RIASSUNTO}

Nonostante le moderne tecniche di conservazione siano in continua evoluzione, i danni di tipo fisiologico e gli attacchi parassitari che si sviluppano durante questo periodo sono ancora piuttosto ricorrenti, causando ingenti perdite economiche. La miglior strategia da attuare per contrastare questa situazione consiste nella prevenzione delle patologie che si potrebbero manifestare in pre- e in post-raccolta. A tale proposito, nel 2015 è stato avviato un progetto triennale finanziato dal programma EU Interreg V "Alpenrhein, Bodensee, Hochrhein", al quale ha partecipato un team di esperti ricercatori provenienti da diversi istituti di ricerca tedeschi, svizzeri e italiani. Il team ha collaborato per riconoscere e classificare le diverse alterazioni che si manifestano prima e durante la conservazione, sviluppando un software di semplice utilizzo e comprensione: Frudistor (da 'fruit disorders storage'). Questa applicazione è comodamente accessibile da qualsiasi dispositivo informatico (PC, tablet o smartphone) digitando http://www.frudistor.de. Tramite un sistema di filtri è possibile selezionare il tipo di danno della mela, la zona del frutto interessata, il momento dell'infezione e consultare la relativa scheda tecnica dotata di svariate immagini illustrative e dettagliate informazioni sulle possibili cause, sintomi e strategie di prevenzione. Attualmente Frudistor contiene più di 40 schede tradotte in italiano, tedesco, olandese e inglese. L'idea di creare un'applicazione accessibile a tutti, gratuita, dinamica ed espandibile, è nata dal desiderio di supportare gli utenti (produttori, magazzinieri e consumatori), a rendere sempre più efficiente e sostenibile il processo di raccolta e conservazione. La seguente relazione raccoglie i risultati di questo lavoro, presentando alcuni dei principali danni che si sviluppano durante la conservazione. 


\section{DANK}

Wir möchten uns bei allen Autoren für die gewinnbringende und konstruktive Zusammenarbeit bedanken. Die Realisierung der geleisteten Arbeit wurde im Rahmen des EU-Programms INTERREG $V$ „Alpenrhein, Bodensee, Hochrhein" ermöglicht, wofür

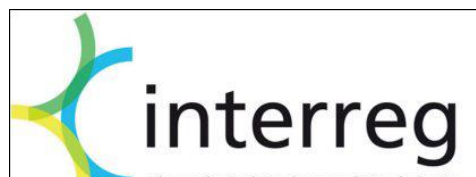

Alpenrhein I Bodensee I Hochrhein

${ }^{\star}{ }^{\star}{ }^{\star}{ }^{\star}{ }_{\star}^{\star}{ }^{\star}$

EUROPÄISCHE UNION

Europäischer Fonds für regionale Entwicklung 


\section{LITERATUR}

[1] Prunier C., Klein N., Neuwald D. (2017). Vom dunklen Herz der Äpfel - Ursachen und Symptome von Kernhausbräune. Obstbau 42 (4), 222-223. Retrieved June 15, 2020 from https://www.researchgate.net/publication/320592192 Vom dunklen Herz der Apfel.

[2] Prunier C., Klein N., Neuwald D. (2017). 'Dunkles Herz' bei Äpfeln - Ursachen und Symptome von Kernhausbräune. Besseres Obst 62 (4), 4-6.

[3] Prunier C., Klein N., Neuwald D. (2017). Del cuore bruno nelle mele. Frutta e vite 41 (6), 8-9.

[4] Prunier C., Klein N., Neuwald D. (2017). Vom dunklen Herz der Äpfel. Obstbau Weinbau 54 (4), 20-21.

[5] Prunier C., Klein N., Neuwald D. (2017). Vom dunklen Herz der Äpfel - Ursachen und Symptome von Kernhausbräune. Mitteilungen des Obstbauversuchsringes 72 (8), 233234.

[6] Prunier C., Klein N., Neuwald D. (2017). Lagerschäden bei Äpfeln - Kernhausbräune. Schweizer Zeitschrift für Obst- und Weinbau 153 (10), 19.

[7] Stürz B., Rossi O., Zanella A. (2017). Wenn die Fruchtschale der Äpfel im Lager verbräunt Schadbilder, Ursachen und Vorbeugung. Obstbau 42 (5), 283-284.

[8] Stürz B., Rossi O., Zanella A. (2017). Wenn die Fruchtschale der Äpfel im Lager verbräunt Schadbilder, Ursachen und Vorbeugung. Besseres Obst 62 (5), 8-10. Retrieved June 15, 2020 from http://kp.eufrin.eu/filead$\mathrm{min} /$ user upload/documents/4844fad9f7234988d9c7f7602040d6eac56.pdf.

[9] Stürz B., Rossi O., Zanella A. (2017). Quando la buccia delle mele scurisce in cella. Frutta e vite 41 (6), 10-12

[10] Stürz B., Rossi O., Zanella A. (2017). Wenn die Fruchtschale der Äpfel im Lager verbräunt - Schadbilder, Ursachen und Vorbeugung. Obstbau Weinbau 54 (5), 13-15.

[11] Stürz B., Rossi O., Zanella A. (2017). Wenn die Fruchtschale der Äpfel im Lager verbräunt - Schadbilder, Ursachen und Vorbeugung. Mitteilungen des Obstbauversuchsringes 72 (7), 215-216.

[12] Stürz B., Rossi O., Zanella A. (2017). Lagerschäden bei Äpfeln - Fruchtschalenverbräunung im Lager. Schweizer Zeitschrift für Obst- und Weinbau 153 (22), 21.

[13] Klein N., Prunier C., Neuwald D. (2017). Glasigkeit bei Äpfeln - Des Einen Segen ist des Anderen Fluch... Obstbau 42 (6), 358-359. Retrieved June 15, 2020 from https://www.researchgate.net/publication/320592380 Lagerschaden bei Apfeln Glasigkeit.

[14] Klein N., Prunier C., Neuwald D. (2017). Glasigkeit bei Äpfeln - des Einen Segen ist des Anderen Fluch. Besseres Obst 62 (6), 17-18.

[15] Klein N., Prunier C., Neuwald D. (2017). La vitrescenza delle mele. Frutta e vite 41 (6), 34-35.

[16] Klein N., Prunier C., Neuwald D. (2017). Glasigkeit - des einen Segen ist des Anderen Fluch. Obstbau Weinbau 54 (6), 18-19.

[17] Klein N., Prunier C., Neuwald D. (2018). Glasigkeit - des Einen Segen ist des Anderen Fluch. Mitteilungen des Obstbauversuchsringes 73 (1), 23-24.

[18] Klein N., Prunier C., Neuwald D. (2017). Lagerschäden bei Äpfeln - Glasigkeit. Schweizer Zeitschrift für Obst- und Weinbau 153 (9), 22.

[19] Kittemann D., Weinmann E. (2017). Schrumpfen (Welken) von Äpfeln im Lager Schadbilder, Ursachen und Vorbeugung. Obstbau 42 (7), 404-405.

[20] Kittemann D., Weinmann E. (2017). Schrumpfen im Lager: Schadbilder, Ursachen \& Vorbeugung. Besseres Obst 62 (7), 19-21.

[21] Kittemann D., Weinmann E. (2017). II raggrinzimento delle mele in conservazione. Frutta e vite 41 (6), 31-33.

[22] Kittemann D., Weinmann E. (2017). Schrumpfen von Äpfeln im Lager. Obstbau Weinbau 54 (9), 23-25.

[23] Kittemann D., Weinmann E. (2018) Schrumpfen (Welken) von Äpfeln im Lager. Mitteilungen des Obstbauversuchsringes 73 (2), 57.

[24] Kittemann D., Weinmann E. (2018). Lagerschäden bei Äpfeln - Schrumpfen (Welken) von Äpfeln im Lager. Schweizer Zeitschrift für Obst- und Weinbau 154 (1), 27.

[25] Köpcke D. (2017). Schalenflecken an 'Elstar'. Obstbau 42 (8), 476-477.

[26] Köpcke D. (2017). Schalenflecken an Elstar. Besseres Obst 62 (8), 19-20.

[27] Köpcke D. (2017). Macchie sulla buccia di Elstar. Frutta e vite 41 (6), 34-35.

[28] Köpcke D. (2017). Schalenflecken an Elstar. Obstbau Weinbau 54 (9), 26-27.

[29] Köpcke D. (2017). Schalenflecken an Elstar. Mitteilungen des Obstbauversuchsringes 72 (5), 151-152. Retrieved June 15, 2020 from http://www.esteburg.de/v1/uploads/Lagerung/Schalenflecken.pdf.
[30] Köpcke D. (2018). Lagerschäden bei Äpfeln Schalenflecken bei Elstar. Schweizer Zeitschrift für Obst- und Weinbau 154 (3), 17.

[31] Stürz B., Rossi O., Zanella A. (2017). Druckstellen - Ein Schaden, der unter die Haut geht. Obstbau 42 (9), 540-541.

[32] Stürz B., Rossi O., Zanella A. (2017). Druck stellen - Ein Schaden, der unter die Haut geht. Besseres Obst 62 (9), 8-9.

[33] Stürz B., Rossi O., Zanella A. (2018). Le ammaccature sulle mele. Frutta e vite 42 (1), 27-28.

[34] Stürz B., Rossi O., Zanella A. (2017). Druckstellen - ein Schaden, der unter die Haut geht. Obstbau Weinbau 54 (10), 24-25. Retrieved June 15, 2020 from https://www.google.com/url?sa=t\&rct=j\&q $=\&$ esrc $=s \&$ source $=$ web $\& c d=8 \&$ cad $=r j a \& u a c$ $=8 \&$ ved $=2$ ahUKEwikwZGhrIPnA hUx0aYKHai6BJMQFjAHegQIBBAC\&url=http\%3A\%2F\%2Fwww.laimburg.it\%2Fen\%2Fprojects-publications\%2Fpublications.asp\%3Fsomepubl_action\%3D300\%26somepubl image id\%3D477259\&usg=AOvVaw2r LKCjCknXVC7oycrnpOJh.

[35] Stürz B., Rossi O., Zanella A. (2017). Druckstellen - Ein Schaden, der unter die Haut geht. Mitteilungen des Obstbauversuchsringes 72 (10), 309-310.

[36] Stürz B., Rossi O., Zanella A. (2018). Lagerschäden bei Äpfeln - Druckstellen: Schäden, die unter die Haut gehen. Schweizer Zeitschrift für Obst- und Weinbau 154 (3), 16.

[37] Köpcke D. (2017). Weiche Schalenbräune. Obstbau 42 (10), 588-589.

[38] Köpcke D. (2017). Weiche Schalenbräune. Besseres Obst 62 (10-11), 19.

[39] Köpcke D. (2017). Riscaldo molle. Frutta e vite 41 (6), 36-37.

[40] Köpcke D. (2017). Weiche Schalenbräune. Obstbau Weinbau 45 (11), 24-25.

[41] Köpcke D. (2017). Weiche Schalenbräune. Mitteilungen des Obstbauversuchsringes 72 (11), 330.

[42] Prunier C., Klein N., Neuwald D. (2017). Fehlender Schalenglanz - Trübschaligkeit oder Weißer Hauch?. Besseres Obst 62 (12), 2021.

[43] Prunier C., Klein N., Neuwald D. (2017). Fehlender Schalenglanz - Trübschaligkeit oder Weißer Hauch?. Obstbau Weinbau 54 (12), 28-29.

[44] Prunier C., Klein N., Neuwald D. (2017). Fehlender Schalenglanz - Trübschaligkeit oder Weißer Hauch?. Mitteilungen des Obstbauversuchsringes 72 (12), 391-392. 
[45] Prunier C., Klein N., Neuwald D. (2018). Lagerschäden bei Äpfeln - Fehlender Schalenglanz - Trübschaligkeit oder Weißer Hauch?. Schweizer Zeitschrift für Obst- und Weinbau 154 (4), 22.

[46] Kittemann D., Weinmann E. (2017). Altersfleischbräune und CA-bedingte Fleischbräune an gelagerten Äpfeln. Obstbau 42 (12), 690-691.

[47] Kittemann D., Weinmann E. (2018). Altersfleischbräune und CA-bedingte Fleischbräune an gelagerten Äpfeln. Besseres Obst 63 (1), 23-24. Retrieved June 15, 2020 from https://www.besseres-obst.at/fachartikel/ technik/2017/12/altersfleischbraeuneund ca-bedingtefleischbraeuneangelagertenaepf.html.

[48] Kittemann D., Weinmann E. (2018). Mela: imbrunimento interno da senescenza e da conservazione in AC. Frutta e vite 42 (32), 36-38.

[49] Kittemann D., Weinmann E. (2018). Altersfleischbräune und CA-bedingte Fleischbräune an gelagerten Äpfeln. Obstbau Weinbau 54 (1), 21-23.

[50] Kittemann D., Weinmann E. (2018). Altersfleischbräune und CA-bedingte Fleischbräune an gelagerten Äpfeln. Mitteilungen des Obstbauversuchsringes 73 (3), 113-114
[51] Kittemann D., Weinmann E. (2018). Lagerschäden bei Äpfeln - Fleischbräune. Schweizer Zeitschrift für Obst- und Weinbau 154 (10), 20.

[52] Stürz B., Rossi O., Zanella A. (2018). Aufspringen und mehliger Zerfall am Apfel während der Lagerung. Obstbau 43 (3), 148-149.

[53] Stürz B., Rossi O., Zanella A. (2018). Aufspringen und mehliger Zerfall am Apfel während der Lagerung. Besseres Obst 63 (2), 4142.

[54] Stürz B., Rossi O., Zanella A. (2018). Spaccature da senescenza e disfacimento farinoso della mela. Frutta e vite 42 (4), 25-26.

[55] Stürz B., Rossi O., Zanella A. (2018). Aufspringen und mehliger Zerfall am Apfel während der Lagerung. Obstbau Weinbau 54 (2). 13-14.

[56] Stürz B., Rossi O., Zanella A. (2018). Aufspringen und mehliger Zerfall am Apfel während der Lagerung. Mitteilungen des Obstbauversuchsringes 73 (6), 221.

[57] Stürz B., Rossi O., Zanella A. (2018). Lagerschäden bei Äpfeln - Aufspringen und mehliger Zerfall. Schweizer Zeitschrift für Obstund Weinbau 154 (6), 20.

[58] Klein N., Prunier C., Neuwald D. (2018). Wenn es zu schnell geht - Schalenverätzungen im CA-Lager. Besseres Obst 63 (3), 2122
[59] Klein N., Prunier C., Neuwald D. (2018). Ustioni su buccia in conservazione AC. Frutta e vite 42 (2), 39-40.

[60] Klein N., Prunier C., Neuwald D. (2018). Wenn es zu schnell geht - Schalenverätzungen im CA-Lager. Obstbau Weinbau 54 (3), 21-22.

[61] Klein N., Prunier C., Neuwald D. (2018) Wenn es zu schnell geht - Schalenverätzungen im CA-Lager. Mitteilungen des Obstbauversuchsringes 73 (5), 154

[62] Kittemann D., Weinmann E. (2018). Stippe an gelagerten Äpfeln. Obstbau 43 (4), 232233.

[63] Kittemann D., Weinmann E. (2018). Stippe an gelagerten Äpfeln. Besseres Obst 63 (4), 38-40. Retrieved June 15, 2020 from https://www.besseres-obst.at/ facharti-

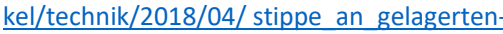
aepfeln.html.

[64] Kittemann D., Weinmann E. (2018). Stippe an gelagerten Äpfeln. Obstbau Weinbau 54 (5), 20-21.

[65] Kittemann D., Weinmann E. (2018). Stippe an gelagerten Äpfeln. Mitteilungen des Obstbauversuchsringes 73 (4), 147.

\section{CC) (1) 8}

Dieses Werk ist lizenziert unter einer Creative Commons Namensnennung-Nicht kommerziell 4.0 International Lizenz.

Quest'opera è distribuita con Licenza Creative Commons Attribuzione - Non commerciale 4.0 Internazionale.

This work is licensed under a Creative Commons Attribution-NonCommercial 4.0 International License.

Für alle Abbildungen und Tabellen ohne Nennung des Urhebers gilt: (C) Versuchszentrum Laimburg.

Per tutte le immagini e tabelle senza menzione dell'artefice vale: (C) Centro di Sperimentazione Laimburg.

For all figures and tables without mention of the originator applies: C) Laimburg Research Centre. 